\title{
Effets de l'encadrement juridique sur la pratique géotechnique
}

F. BLONDEAU

Consuitant

38, rue Boiteau 75016 Paris

S. BOREL

Solétanche-Bachy 6, me Watford 92000 Nanterre

\section{M.L. CARRIÈRE}

Avocate

134, bo Saint-Germain 75006 Paris

\section{P. GUILLERMAIN}

Expert

6, rue Louis-Pasteur 92774 Boulogne-Billancourt

Cedex

\section{J. ROBERT}

Arcadis 9. av. Réaumur 92354 Le Plessis-Robinson Cedex
La recherche du " risque zéro m, le développement de la normalisation, la désignation d'un responsable à tout préjudice [troís caractéristicques de notre société actuelle] s'imposent également à la pratique de la géotechnique dont l'objet d'étude est le sous-5ol par nature même domaine des incertitudes car non visible, hétérogène et evolutif.

L'anglyse des effets de cet encadrement juridique inévitabie conduit à proposer les principales pistes d'amélioration suivantes pour tenjr compte de la réalité géotechnique :

- mettre en place un véritable management des risques à chacune étape du projet, avec transparence, partage. réactivité et traçabilité des décisions influant sur l'évolution du projet;

- favoriser l'implication plus continue du géotechnicien. au cours du projet pour une analyse cohérente et globale des risques géologiques:

- impliquer plus étroitement les assureurs dans ka gestion des risques, afin de développer des programmes d'assurance cohérents et représentatifs du poids économique et des responsabilités réelles des différents acteurs du projet :

- recourir plus fréquemment aux contrats sur prix mixtes forfaitaires et unitaires et développer les contrats en partenariat pour gérer les risques et optimiser le projel dans une relation gagnan1/gagnant entre tous les intervenants ;

- former et sensibiliser les juristes et les ingénieurs aux spécificités de la géotechnique : aspect prototype cle chaque ouvrage, sous-sol hétérogène et évolutif loin des matériaux inđustrjels, normes ne pouvant pas couvrir toutes les situations et devant laisser la possibilité d'innover';

- en cas dé litige, faire jntervenir des experts spécialistes en géotechnique et intéresser effectivement les parties à la résolution des conflits au plus vite et au moindre coût.

Mots-clés : géotechnique, juridique, risques, responsabilité, contrats
NDLR : Les discussions sut cet article sont acceptees jusqu'au 1 mars 2009.

\section{Effects of liability and insurance legal framework on geotechnical practice}

The research of a risk zero in, the development of standardization, the research of a person ljable for any camage (three characteristics of our French Society) have a great influence on the geotechrical practice studying the ground which is ron visible, heterogereous, changing and with uncertainttes. The analysic of this inewitable legal framework effects leads to propose the main following inprovement. actions taking account of geotechnical reality :

- set up a true hsks management at each project stage, with transparency, sharing, reactivity and traceability of the decisions intluencing the project modifications;

- support the more continuous implication of the geotechnical engineering during the project for a coherent and total analysis of the geological risks; 
- imply the thsureps in the risk managyement in nrtar fa tewelng prograros of insurance coherent and representative of the economic weight and real responsibilities for the project actors; - resort to the contracts on mixed fixed and unit prices and develop the contracts in partnership for risks management and project optimizations in a relation gaining/gaining between all the actors :

- train amd sensitize lawyers and engineers with geotechnical speciftcities : each project is unique, grourd is heterogeneous and changing far from industrial matorials, atandards are not able to cover all the situations and have to leave the possibility of innovating:

- in case of litigation, choose experts specialists in geotechnic and interest the actors in the resolution of the conflicts as fast as possible and at a bwer cost.

Key words : geotechnic, legal. risks, liability, contracts.

\section{Objet du document}

Contrairement à ce que lalsse imaginer l'article 1792 du code civil, le géotechnicien dit que le sol n"a pas de « vice $n$. Le sol, c'est-â-dire la nature, est le partenaire obligé du spécialiste. "On ne commande à la nature qu'en lui obéissant $3^{\text {"th }}$ et on ne peut pas tricher" avec elle. Ce constat, dont on ne prend conscience qu'à l'usage et toujours à ses dépens, impose au géotechnicien une humilité permanente dans l'exercice de son talent.

Mais une conduite sage n'est malheureusement pas suffisante pour mettre a l'abri des risques matériels et corporels inhérents aux conséquences des nombreuses incertitudes qui caractérisent notre compréhension très imparfaite des lois de la nature.

La loi du 4 janvier 1978 relative à la responsabilité et à l'assurance dans le domaine de la construction, dite loi Spinetta, et l'ordonnance du 8 juin 2005 a portant modification de diverses dispositions relatives à l'obligation d'assurance dans le domaine de la construction et aux géomètres experts o instituent une assurance obligatoire des risques de la construction dont l'objet est de couvrir la réparation des dommages we nature décennalen des ouvrages de bâtiment et en précisent les conditions d"application.

Ces textes, accompagnés du cortège de documents techniques rationaux ou europèens, à caractère ( indicatif „ ou " normatif ", sont lè pour limiter les risques et protéger les maítres d'ouwage contre les conséquences fấcheuses des interventions des acteurs de la construction. Initialement limités aux u ouvtages de bătiment m, ils ont - par extension prugressive - induil de profondes modifications des conditions de la pratique professionnelle.

Ĺabjet de ce document est d'en analyser les effets - bons ou mauvals - sur l'exercice de la profession de géotechnicien et de proposer des améliorations.

\section{Du constructeur au géotechnicien : vingt siècles de responsabilité}

Dans la première édition de son livre, Jacques Montmerle cite l'époque du roi de Babylone et de Chaldée, Hammourabi (1730-1685 av. J.-C.), quil avait instiłué un régime pour le moins sévère contre les mauvais constructeurs:

a Si la maison construite s'est écroulée et a tué le maître de la maison, l'architecte est passible de mort, si c'est l"enfant du maître de la maison qu'il a tué, on tuera l'enfant de l'architecte... m

uSi c'est la fortune mobilière qu'il a détrutte, il restituera tout ce qu'il a détruit et parce qu'il n'a pas rendu solide la construction et qu'elle s'est effondrée, il restituera la maison à ses propres frals. y

Au temps des Romains, le législateur a institué une responsabilité de quinze années de l'architecte en matière de travaux publics (385 apr. J.-C.., Constitution de Gratien et de Théodosej.

L'ancien droit français a repris l'ancien droit romain et l'a étendu aux travaux privés en réduisant le délai de garantie à dix ans.

Pendant très longtemps, la différenciation entre le maitre d'ceuvre et l'entreprise n'est pas clairement etablie, larchitecte étant souvent un architecte-constructeur:

Lors de la rédaction du code civil ce délai de prescription de dix ans en faveur de l'architecte et de l'entrepreneur, à raison de la garantie des gros ouvrages qu’ils ont dirigés ou faits, est maintenu.

Il faut attendre les progrès techniques considierables du xix siècle pour voir se mettre en place une véritable organisation professionnelle, notamment des entrepreneurs avec la création du Syndicat général de l'entreprise en 1853, puis l'apparition de la Fécération du bâtiment en 1859 .

Cette même année 1859 a vu lá création de la Mutuelle des entrepreneurs.

Pour les architectes, il faut attendre 1931 pour que soit fondée la Mutuelle de l'association provinciaje.

La seconde moitié du xxe siècle est marquée par 
la renommép des rpéatelurs des hureaux d'études de sols qui ont profondément influencé la mécanigue des sols française, tels que Jean Kerisel (SIMECSOL, 1952), Jacques Florentin (MECASOL, 1948), Louts Parez (SOL-ESSAIS, 1953) pour ne citer que ceux qui nous ont quittés.

Les plus jeunes de la profession pourtont se faire tme idée de l'environnement de la praticue géotechnique avant les annees 80 au travers de cet extrait de l'article du juriste Pierre Liochon - à l'époque chef du bureau des études et recherches de la direction du Service national de la protection civile au ministère de l'Tntérieur-publié dans le numéro spécial du Bulletin de haison des laboratoires des ponts et chaussées consacré à la stabilité des talus (1976). L'article portait sur les aspects juridiques des problèmes de glissement's de terrain:

a Les géotechniciens interviennent dans des circonstances tres diverses et três variées et pour des affares d'importance très inégale... II faut d'abord observer que l'intervention d'un géotechnicien n'est pas obligatoire en France dans l'état actuel de la réglementation. D'alleurs, la définition juridique d’un géotechnicien est difficile à préciser : le mot n'est pas protégé, il n'existe pas un ordte des géologues ou géotechniciens pouvant imposer une certaine discipline déontologique ; les filières pédagogiques permettant de s'intiuler géotechniciens sont variés et concurrentes.

Cette absence de reglementation et cette inorganisation juridique favorisent la profession gui connait un grand essor.

"Bien entendu, les maitres d"ouvrage ont toute hiberté pour choisir Jeurs spécialistes des problènes de sol et de sous-sol. Que ce soit l'Étar ou une collectivité locale pour la confection de cartes géotechnicues ou l'étude d'un ouvrage (ponts, barrages, talus, routes et autoroutes), que ce soit un promoteur pour l'anénagement d'une station de sport dhiver ou plus simplement pour la construction d'un immeuble, tous ont recours aux géotechniciens de leurs goúts. C'est celui qui paye qui décide du choix.

"Lambiance psychologique est en général très bonne du fait de la communauté intellectuelle et de la communauté dintérét qui existent entre le géotechncien et les ingénieurs des bureaux d'études dont dispose le maitre de l'ouvage; ces ingénieurs sauront conprendre le langage et les états d'âme du géotechnicien.

"Par aileurs, l'environnement regiementaire est nul. Le géotechnicien est lié au maître de louvrage ou au maitre d"ceure par un contrat qui definit d"une manière plus ou moins précise sa mission. Cette mission peut être linitée à une étude de sol et de sous-sol: dans ce cas, le géotechnicien ne participe pas directement à l'acte de construire, ce a'est pas luj qui ếtudie les fondations et la structure du batiment et de l'ouvage; il se contente, et c'est déjà beaucoup, de donner des élérnents techniques chiffés awx ingénieurs du bureau d'etudes et de contrôle. Cependant, on peut noter une évolution vers l'élargissement progressif de la mission du géotechnicien vers la conception et le dimensionnement des ouvrages (fondations, soutenements, talus, etc.); cette évolution dépend principalement de la personnalité même de l'ingénieur-consell, de la façon dont il comprend son role, de la profondeur de ses connaissances et de son goút pour les responsabilités. Jusqu'à présent, il semble qu'aucune jurisprudence n'ait établi la responsabilité du géotechnicien au sens strict pour son étude de sol. Dans les sinistres qui auraient pu fare jurispru- dence. le géotechnicien au sens larae létude de sol et conception) était confondu avec les ingénjeurs dans un bureau d'études de calcul d'ouvrages.

"De toutes facons, le gétechnicien doin tenir son róle avec franchise. La géotechnique a des limites; c"est une science expérimentale qui évolue en permanence et non une technique éprouvée reposant sur des règles strictes. Alors que le béton et le métal se calculent selon des règlements précis, la géotechnique est encore un art. Le géotechnicien ne doit donc pas bluffer; il doit employer avec pondération le conditionnel et les formules dubitatives ou restrictives, mais il ne doit pas non plus chercher à tout prix a coumrim sa responsabilité en exigeant des mesures longues ef coûteuses, ou en inposant des dispositions constructives manifestement exagérées compte tenu des ristutues présentés par l'ouvrage. C'est un jeu difficile où la vie de nombreuses personnes et des biens considérables peuvent être mis en cause. y

Heureuse époque oủ l'ambiance était effactivement exempte de la crainte d'une o mise en cause La faiblesse des hypothèses et des modêles que l'on śautorisail à développer selon l'adage de Karl Terzaghi " learn as we go a appliqué ane discipline encore balbutiante, ne se heurtait pas encore au diktat des kilos de papier débités par l’informatique omniprésente, sensée formaliser la science légale.

Dans les annees 70, période faste pour les concepteurs et constructeurs d'autoroutes, les études de stabilité de pentes se faisaient essentiellement à l'aide d’abaques et les premiers logiciels de calcul développés au Laboratoire central des ponts et chaussées étaient résemés à l"usage du noyau de spécialistes du rëseau des LPC, de crainte de voir l'infomatique prendre le pas sur la réflexion chez des ingénieurs que pouvait fasciner l'apparente magie de ce nouvel instrument de travail. De memoire, les cas de glissements n'étaient pas plus spectaculaires qu'aujourd"hui et les coûts de confortements préventifs restaient raisontables.

La praticue de la géotechnicue faisait traditionnellement appel à deux domaines de compétences:

la théorie acquise dans les diverses filières d'enseignement de l'ingénterje - écoles d’ingénieurs, facultés de génie civil. IUT - elle est indispensable pour comprendre les fondements géologiques, hydrauliques, minéralogiques et mécaniques de la discipline; mais, depuis que l'a souligné P. Liochon dans ses écrits de 1976, la définition juridique d"un géotechnicien, c'est-à-dire des compétences qui lui sont nécessaires, n'est toujours pas établie en 2008 :

- lexperience des auvages construits : acquise avec le temps, beaucoup de temps, elle est le conplément indispensable qui permet d'intégrer la prise en compte des écarts systématiques entre le comportement a sur le papier w et le comportement « sur le terrain n. Dans son discours prononcé à l'occasion de linauguration de l'amphithéâtre Albert Caquot, le 25 mai $1977^{(2)}$ Roger Coguand (IGPC) retraçait les grandes étapes de la vie professionnelle de celui qui fut certainement l'un des plus grands ingénieurs de génie civil du $\mathrm{XX}^{e}$ siècle et soulignait de son cuvre et de ses

(2) to Hommage à Albert Caquot n, ENPC.. 
puhitications que * Toutes ces innnyations ont été inspirées par l'expérjence. Dans la préface de louvrage (Équilibre des massifs à frottement interne. Stabilité des terres pulvérulentes et cohérentes, 1934), lauteul écrit": "Les ingénieurs ne doivent pas oublier qu'ils sont avant tout des physiciens" - critique implicte des théories mathématigues trop abstraites ne se prêtant pas à des procédés de mesure commodes et fidèles. La démarche intellectuelle d Albert Caquot est toujours la même. Mis par son expórience pratique en face d'un probleme, son intuition aiguë lui en fait pressentir une solution. que sa maitrise des sciences physiques ef mathématiques lui permet de demontrer. y

Depuis ces années techniques, le droit s'est invité d'office à la table de travail du géotechnicien, en la personne de son avocat. Ces deux partenaires ne parlent pas la même langue.

\section{3}

\section{Le cadre juridique actuel}

Ce bref rappel du cadre dans lequel s'inscrit la responsabilité du géotechnicien est largement inspiré de deux documents rédigés par $\mathrm{M}^{\mathrm{e}}$ Marie-Laure Carrière :

- " Les obligations des bureaux d'études de sols $x$, rapport pour l'USG, jutin 1996 ;

- « La responsabilité en matière de sol w, colloque Jus. tice et Construction, Paris 2004;

ainsi que d'informations recueillies sur le site internet de la SMABTP(3)

\section{1}

\section{Les principes généraux de la responsabilité}

L'objet de la responsabilité civile est la réparation d’un dommage, alors que la responsabilité pénale constitue la sanction d'une infraction.

La responsabilité civile impose de réparer le dommage causé à autruj et suppose en principe l'existence de trois éléments:

- une faute:

- un dommage :

un rapport de causalité entre ces deux élémenls.

Les deux régimes de responsabilité civile délictuelle et contractuelle de droit commun coexistent et se distinguent dans la rédaction du code civil :

- la responsabilité délictuelle, qui est l'obligation. pour l'auteur d'un fait donmageable, d'indemniser le tiers victime. Elle est fondée sur les articles 1382 et suivants du code civil, les juges apprécient le comportement et qualifient la faute selon sa gravité. En dehors de tout contrat, l'action s"exerce nécessairement sur le fondement délictuel et en dehors de cas de présomption de responsabilité, la charge de la preuve repose sur la victime:

[3] http:/Www.smabtp.fr/MebCommu/WEB_P_GENERALE.NSF/0/ 72478D E30FBSB82FC125706E003B310F? OpenDocument
- la responsabilité contractuelle, cui est l'obligation. pour le contractant qui ne remplit pas une obligation prévue au contrat, de réparế le dommage causé à l'autre partie. Ĺobligation est appréciée au regard de la convention liant les parties et donc de l'étendue des obligations. C'est dans ce domaine qu'est née la distinction jurisprudentielle entre les obligations de moyens et les obligations de résultat. Le point essentiel est de déteminer sum qui repose la charge de la preuve.

En maliẻre de construction, en matière contractuelle à l'égard du maître d'ouvage, deux régines de responsabilité peuvent s'appliquer

- la responsabilité de droit commun, dont sont débiteurs les constructeurs à l'égard du maitre de l'ouvrage avant ou en l'absence de réception. ou pour les dommages qui ne sont pas de nature décernale. Elle s’appule sur les principes gếnéraux évoqués ci-dessus:

- la responsabilité dite a légale n (loj Spinetta\}. à savoir les garanties décennale, biennale et de parfait ackevement, qui implique une présomption de responsabilité des constructeurs, après réception, potr une durée déterminée de 10 ans à compter de la réception de l'ouvrage, rais seulement pour les dommages graves entrant dans le champ de ces garanties.

\section{2}

\section{L'obligation de moyens}

Se fondant sur la notion d'aléa, la doctrine a distingué: - l'obligation de résultat, dont l'objet est strictement déterminé : elle fait reposer sur le contractant une présomption de faute, dont i] pourrá s'exonérer par la démonstration d'une cause étrangère. C'est une responsabilité de plein droit, telle que celle imposée par. la loi Spinetta

- l'obligation de moyens, ou de prudence et diligence - elle n'engage le débiteur qu'à un certain comportement, sans garantie de résultat. C'est précisément le cas du médecin, qui doit prodiguer des soins consciencieux, conformes aux doninées acquises de la science, mais ne peut s'engager à guérir ses malacles. Cette obligation n'est sanctionnée que si tous les moyens promis ou escomptés raisonnablement n'ont pas été mis en oeuvre et le débiteur de l'obligation peut être dégagé de toute responsabilité en prouvant qu'il s'est comporté wen bon père de famillew selon les prescriptions de l'article 1137 du code civil.

Les juges apprécient la nature et J'étendue de l'engagement pris en fonction de cette distinction et déterminent ainsi qui supporle la chatge de la preuve. A titre d'exemple, ils font reposer une obigation de résultat sur l'entrepueneur qui s'oblige à construire un imneuble suivant un plan déterminé moyernant un prix convenu dans un certain délai. En revanche, its n'imposent qu'une obligation de moyens pour des prestations d"études tendant à pawenir á un résultat reconnu incertain

Si un se félère à l'aléa, l'étude de sols constatue en priacipe une obligation de moyens. La faute du géotechnicien doil donc étre prouvée.

En fait, il existe une multitude de gradations des obligations de movens et de résultat et certaines 
obligations comportent les deux caractères soit simul tanément, soit successivement. Ainsi, la qualification d'obligation de moyens des bureaux d'études de sols trouve ses limites dans l'engagement des parties et l'état actuel de la science. A titre d"exemple, le respect des délais, l'exécution du nombre de sondages prévus, à l'emplacement fixé, la véracité des caractéristiques des terrains rencontrés au droit des sondages, sont autant d'obligations finalement de résultat.

L'important est d'exécuter les obligations conformément aux règles établies, ce qui conduira nécessairement à atteindre les objectifs fixés, en l'absence d'aléa et il est donc nécessaire de démontrer le caractère aléatoire de certaines techniques, mais après avoir mis en ceuvre tous les moyers possibles pour parvenir à un résultat.

En matiêre de construction, la prewve est gẻnéralement établie par le biais d"une expertise judiciaire ordonnée en vertu de l'article 145 du nouveau code de procédure civile.

Dans ces conditions. c'est un expert technique qui appréciera en premier lieџ si le debiteur d'une obligation a fait preuve d'une diligence et dune compétence normale eu égard à l'état actuel de la science, s'il a respecté les normes et usages de la professton et s'il a rendu un service conforme à ce que le bénéficiajre pouvaít normalement attendre d'un tel professioniel.

L'enjeu des expertises judiciaires est donc très important puisqu'elles assument la charge de la preuve et conditionnent' l'appréciation ultérieure des juges.

Ansi, l'intérèt de la théorie distinguant les obligations de moyens ou de résultat s'efface devant la nécessité, surtout pour une science en évolution telle la géotechnique, de recourir à une expertise cui étabit, avant toute décision sur les responsabilités, la faute du spécialiste du sol, au regard des règles de l'art.

Les tribunaux entérinent souvent les conclusions de l'expelt, même si ce dernier a effectué une mauvaise appréciation des données technjques d'une matière dépassant ses propres compétences. Pourtant, le caractère aléatoire de la géologie et les limites scientifiques auxquelles se heurtent les gêtachniciens doivent conduire les tribunaux à définir strictement les obligations contractuelles des bureaux d'études suivant chaque cas d'espèce, afin de ne pas reporter le risque du sol sur ces intervenants et bouleverser l'économie du contrat (honoraires dérisoires au regard du coût global de la construction).

La nomalisation constitue alors wn garde-fou indispensable contre les abus tendant à faire supporter aux professionnels, par l'extension de la notion de faute, la charge de sinistres résultant parfois simplement de contraintes financières.

\section{3}

\section{Le devoir de conseil}

Lobligation de conseil, et plus généralement d'information, est une création jurisprudentielle, devenue un fondement juridique autonome suffisant a une action en justice. Imposée au titre d"un contrat. la base juridique de cette obligation semble être l'article 1135 du code civil, gui oblige les contractants non seulement au respect des stipulations explesses.

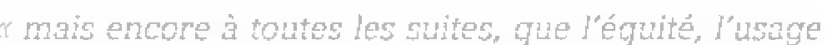
ou la loi donne à l'obligation d'après sa nature o, mais la jurisprudence se réfêre égajement aux vices du consentement, nolamment l'article 1116 relatif au dol, à l'article 1147 ou même à une prétenclue obligation précontractuelle fondée sur" l"article 1382

En matière de construction, on note une évolution importante dans lapplication du devoir de conseil. Cette notion recouvre le devoir de conseiller la solution la plus favorable, d'informer des contre-indications de sa propre prestation, do ses limites, des risques elacolrus, des contraintes. Létendue de l'obligation varie en fonction du contrat lant le constructeur au maitre de louvrage, professionnel avisé ou profane, assisté ou non d'un conseil professionnel. L'absence de maitre d'ceuve doit conduire ane plus grande vigilance.

Face à un maitre d'ouvrage inexperimenté, l'obligation du bureau d'études détenant la compétence est plus lourde et en matière de géotechnique, il est rare de voir le devoir de conseil s'effacer devant la compétence notoire du maître de l'ouvage, cause exonératoire de responsabilité. Il ne reste alor's que la force majeure. Le géotechnicien subit donc une présomption irréfragable de connaissance de l'information dès lors que celle-ci releve de ses compétences.

Dans le cadre des prestations d'un bureau d'études, le consell constitue souvent l'obligation principale. Mais lorsque la jurisprudence condamne sur le fondement du devoir de conseil, il ne s'agit pas de sanctionner l'inexécution de cette obligation, mais d'un devoir accessoire hors prestations.

Ainsi en principe, le devoir de conseil impose au géotechnicien de signaler les risques de faits portés à sa connaissance, mais dont il ne supporte pas la responsabilité principale, parce qu'ils sont exclus du champ strictement contractuel.

Il existe parfois une confusion, parce qu'il est difficile pour un juge de délimiter précisément le cadre contractuel dans un domaine technique.

En définitive, le juge interdit toute attitude passive des constructeurs et favorise une veritable immixtion dans des décisions qui ne leur incombent pas normalement. Cela entraine un enchevêtrement des missions et compétences, pour parvenil à une dilution des responsabilités.

Face à cette situation, l"assureur donne les conseils sulvanls:

"Lorsque des "variantes" sont sollicitées par le client, adressez-lui par écrit wos remarques sur Jes solutions adoptées. Attention, cependant, ž ce que cette mise en garde ne conclut pas an risque certain de survenance de désordres, auquel cas, en l'absence d'aléa, l'assurance ne serait plus possible! Alertez le client sur les éventuelles limites ou contraintes d'utilisation de louvrage réalisé : charges admissibles, ouvrages non circulables, etc. Veillez à remettre au client, lors de la livraison, un document précisant clairement les modalités d'entretien ou de maintenance de l'ouvrage ; le cas échéant, attirez son attention sur la nécessité de conclure un contrat d'entretien. N'oubliez pas que, mẻme si certains DTU prévoient expressément les modalités d"entretien de l'ouvrage (DTU Étanchéité, par exemple), les dispositions de ces documents ne s'imposent pas au client : c'est à l'entrepreneur d'en rappeler la teneur auprès de son client. w 


\section{La loi Spinetta}

Lobjectif de la loi $78-12$ du 4 janvier 1978 , dite loj Spinetta, est de protéger le maître de l'ouvrage des conséquences des sinistres par ume modification du régime de responsabilité, une implication du monde de l'industrie et une réforme de l'assurance construction, obligatoire pour les travaux de bàtiment, avec un systeme de préfinancenent de l'assureur dommage-ouvrage. Elle donne une protection au maitre de l'ouvrage, tout en limitant la responsabilité des constructeurs aux dornages, même résultant d'un vice du sol, qui compromettent la solidité de l’ouvrage ou le rendent impropre à sa destination. Les princlpales dispositions de cette Iot, relatives à l'objet de la présente analyse, sont rappelées en annexe.

Pour les marchés publics, sans reprendre les dispositions littérales du code civil, le juge administratif refient en la matiëre les principes dont s'inspirent les articles 1792 et 2270 du code civil et fait directement référence à la lol du 4 janvier 1978

La responsabilité décennale trouve donc application en droit public comme en droit privé pour tous les dommages graves des ouvages de båtiment et de gérie civil.

Cortelativement ta loi comporte un volet assurance dont les conséquences sur l'exercice de la profession sont considérables et seront commentées ci-après.

Il est important de commenter ici les situations visées par la loi et précisées par l"ordonnance 2005-658 du 8 juin 2005.

- Constructeur. La loi s'applique aux seuls " constructeurs de l'ouvrage n que l"article 1792-1 du code civil definit ainsi : Tout architecte, entrepreneur, technicien ou autre personne liée au maitre de louvrage par un contrat de louage d'ouvrage... n.

Ce régime est donc applicable au bureau d'études de sols, lonsque ce derner intervient, ce qui est souvent le cas, pour le compte du maitre de l'ouvrage La responsabilité contractuelle de droit commur reprend en revanche son office dans le cas d'une intervention du géotechnicien en qualité de soustraitant du maitre d'ceuvre ou die l'entreprise.

- Ouvrage. La notion d'ouvrage désigne la globalité d'une construction de caractère immobilier, ainsi que tous ses éléments constitutif's et d'équipement indissociables.

- Batiment. Dans son texte initial, la loi Spinetta circonscrit !'obligation d"assurance aux « travaux de

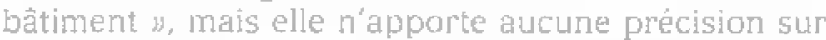
les ouvrages concernés par cette notion. A défaut d'une telle définition, on a assisté à une extension jurisprudentielle de son champ d’application, les tribunaux allant jusqu'à inclure certains ouvrages de génie civil dans le champ d'applicution de l"assurance obligatoire. Pour rétablir l'esprit de la loi, le Gouvernement a pris l'ordonnance du 8 juin 2005 a portant modification de diverses dispositions relatives à lobligation d'assurance dans le domaine de la construction et aux géométres
Pxnerts n nuli s'insniro dı mapport déposé le 18 décembre 1997 par trois éminents juristes, dont $M^{k-} J$.-P. Karila qui en a détaillé les dispositions dans un article du Moniteur auquel nous renvoyons le lecteur ${ }^{14 "}$. Ainsi. cette ordonnance

- définit le champ de lassurance obligatoire, en établissant la liste des ouvrages exclus de son champ d'application, notamment les ouvrages de génie civil ou infrastructures

exclut également du champ d'application de l'assurance obligatolre les éléments d'équipenent dont la fonction exclusive est de permettre l'exercice d’une activité professionnelle.

Sous réserve d'inventaire, le champ d"apolication de l'assurance obligatolre défini par ces dispositions de l'ondonnance est cohérent avec celui que précisait la circulaire du $79-38$ du 5 avil 1979 du Ministre de l'Envijonnement ét du cadre de vie pouti" les marchés publics.

- Assurance des dommages aux existants. Les imprécisions du texte initial on conduit la jurisprudence à opérer, la encore, une interprétation extensive de la loi concernant I'assurance des dommages aux existants. D'une façon générale, les tribunaux ont en effet adnis que lorsque l'on ne pouvait dissocier les existants des travaux neufs (incorporation de l'existant à l'ouvrage neuft, il converait de leur appliquer l'assurance obligatoire. Lordonnance de 8 juin 2005 est venue préciser ce point

- l'obligation d"assurance re s'applique pas aux ouvrages existants avant l'ouverture du chantier. à l'exception de ceux qui, totalement incorporés dans l'ouvrage reuf, en deviennent indivisibles;

pour les dommages causés aux existants qui ne seraient pas incorporés aux travaux neuf́s (existants physiguenent êloignés, par exemplej, l'assurance obligatoire ne s'applicue donc pas.

- Délai de prescription pour les sous-traitants. Par principe, le sous-traitant n'est pas soumis à l'obligation d"assurance décennale. En effet, tiers au marché principal entre l'entrepreneur et le maitre d'ouvrage, il n'engage que sa responsabilité contractuelle envers l'entrepreneur principal qui lui confie le marché de sous-traitance, et sa responsabilité délictuelle envers le maitre d'ouvrage. Le sous-traitant est donc soumis à une prescrjption a de droit commun $n$ qui, en ce qui conceme le géotechnicien, í impose en principe une "obligation de moyens is commentée précédemment. Dans ce cadre, sa responsabilité court à compter de l'apparition du dommage alors que celle de la responsabilité clécennale court ả compter de la réception des travaux. Afin de garantir une sécurité juridique aux sous-traitants, l'urclunnafice prévoil désurmals que les actions au titre des désordres de nature décennale mettant en cause la responsabilité des sous-traitants sont soumises aux mênes délais de prescription que celles qui mettent an cause la responsabilité des constructeurs. La prescription de ces actions est donc de 10 ans, à compter de la réception de l'ouvrage.

(4) u Responsabilité assurance constructon : la réfome du 8 juin

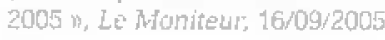


- Cas d'exanération de responsabilité En cas de dommages d'ordre décennal, les constructeurs sont responsables de plein droit, ce qui dispense le maître de l'ouvrage de la démonstration d'une faute. Les juges font une application tres stricte de ce principe d'ordre public. Toutefois, l'alinéa 2 de l'article 1792 prévoit a Une telle responsabilité n'a point lieu si le constructeur prouve gue les dommages proviennent d'une cause étrangère. w Il existe deux cas de cause étrangère :

- la force majemre. Elle suppose réunis les trois carac tères d'irrésistibilité, d'imprévisibilité et d'extérioritê. Elle est rarement admise ;

- le fait du maitre de l'ouvrage. La faute du maître de I’ouvrage revêt principalement trois aspects

- l'immixtion dans le processus de construction d'un maitre d'ouvrage notoirement competent ;

- l"acceptation délibérée du risque par un maître d'ouvrage dûment informé, par écrit, des limites et risques présentés par ses choix constructifs (économie abusive, par exemple) ; il semble en effet nomal que le maître d'ouvrage soit responsable du choix qu'il impose, dès l'instant où ce dernier" a été fait en toute connaissance de cause:

- la mauvaise utitisation de l'ouvrage ou son défaut d'entretien, à la condition que le constructeur démontre qu'il a rempli son devoir de conseil en attirant l'attention du maître d'ouvrage sur les limites d'utilisation ou les contraintes d'entretien de l'ouvrage réalisé.

\section{4}

\section{L'environnement du géotechnicien}

Le géotechnicien intervient dans un contexte où sont implicués différentes parties dont chacune doit jouer son rôle dans le cadre de ses engagenents contractuels.

\section{1}

\section{Le maître d'ourrage}

Il s'en remet à son maitre d'ceuvre pour les décisions techniques. Etant observé que, dans le domaine du batiment, à la différence des carrelages de salle de bain, les fondations, les ouvrages de soutènement et les éventuels ouvrages hydrauliques ne sont en rien un argument de commercialisation de l'ouvrage acheté. Le géotechnicien apparaît comme un facteur de dépenses inutiles auquel on n'a aucun scrupule a imposer en permanence une contrainte de coûts qui a forcément une conséquence sur la qualilé de la prestation founie et augmente les risques d'être impliqués dans de futurs désordres.

On imagine mal un patient dictant au médecin la liste de ses prescriptions. Un maitre d'ouvrage ne voit pas d'inconvénient à dicter ou à faire dicter sa conduite au géotechnicien. car, dès lors qu'il est entouré de prestataires assurés, il est imperméable à toute argumentation visant à lui démontrer qu’il est de l'interèt de tous de solgner les études de ce qui va être la base sur laquelle va repuser lout son ouvrage plutôt que de reprendre en sous-ceuve des fondations qui risquent de s'avérer inappropriées faute d’études suffisantes.

\section{Le maître d'œuvre}

Pris en tenaille entre les exigences financières du maître d'ouvrage et ses propres impếratifs techniques, il a tendance à se décharger $54 r$ le géotechnicien de toute responsabilité eu égard aux conséquences du comportement des parties d'ouvrage en contact avec le sol, tout en bridant les demandes légitimes du géotechnicien retenu, quil finira par accepter d"intervenir a sans filet $y$ et sans assurer l'accompagnement géotechniçue du projet pourtant prévu par la norme sur les missions d'ingénierie géotechnique.

\section{3}

\section{Le sol}

Il est incontournable. Ses propriétés n'ont pas été affectées par la loi Spinetta. Les essais en laboratoire ou en place sont quasiment les mêmes qu'il y a cinquante ans. Seul le développement de l'infomatique permet de progresser si et selilement si les hypothèses introduites dans les modèles sont fiables "cest-à-dire jssues de bonnes mesures ou de calages appropriés. Sur ce thème, les trente demières années ont vu un bouleversement des objectifs à atteindre : d'une mécariçue de la rupture, plus immédiatement à la portée de calculs "manuels $n$, on est passé à une mécanique des déformations permise par l'informatique et supposée rếpondre à la demande toujours plus pressante des maitres d'ceuvre de réduire les déplacements des structures. L'interaction sol-structure a déplacé sur le sol, qui est l'élément le plus complexe à modéliser, la responsabilitê de ne pas solliciter les structures au-delá du domaine de déformation autorisé par les règlements y afférents. Il est loin le temps où. demandant au géotechnicien de limiter à moins d"un centimètre par an sur dix ans les tassements d"une plate-forme de triage reposant sur $10 \mathrm{~m}$ de tourbe franche, le géotechnicien, avisé et conscient des limites de sa discipline, a pu convaincre le client qu'il était plus sage d'adapter les perfomances du futur dispositif ce freinage automatique des wagons aux inévitables tassements du sol plutôt que le contraire.

A titre d'illustration, il est bon de souligner que les usages en matière de reconnaissances de sols conduisent à préterdre caractériser l'emprise des fondations d'un projet sur la base d’un échantillonnage qui, dans les cas faworables, équivaut à voutoir caractériser toute la population française en interrogeant moins de cing individus.

\section{4}

\section{L'entreprise}

Lorsqu"elle connait le sol - terrassier, entreprise de fondations spéciales ou de travaux souterrains l'entreprise est, comme le géotechnicien, tenue d'un devoir de conseil. L'entreprise doit fréguentinent adapter ses méthodes d'exécution à des situations inattendues, à un sol dont les caractéristiques sont parfois très différentes de celles annoncées, avec le souci constant de terminer son ouvrage et de ne pas créer de situations provisoires ou définitives incompatibles avec les hypothèses justificatives du dossier. 


\section{L'assureur et le contrôleur technique}

L'assureur est l'interlocuteur caché mais omniprésent dans les esprits. C'est, avant tout, un financier qui n'a que très peu de préoccupations techniques dès lors que le montant des primes couvre le montant des sinistres à vernir. La prevention n'est pas son objectif premier et le bonus n'existe pas dans ce domaine alors qu'on pourait inaginer que le contreseing d'un avocat au bas d'un contrat soit un gage de sécurité qui apaise les craintes de l'assureur lequel en tient compte dans le calcul de ses primes.

Comment peut-il être rassuré sur le bon déroulement du projet quiil assure, si le contröleur technique. qui est son homme de l'art sur le terrain, est dans l'incapacité d'exercer sa mission sérieusement faute d'être rémunéré ả juste prix par un maître d"ouvrage qui l'a mis en concurrence au même titre que tous les autres corps de métier. Seul un retour à une rémunération cirecte et adéquate des contrôleưs par les assureurs permetrait de rendre au contrôle son sers at de limiter les sinistres.

Mais est-ce un objectif de l'assurance ? On constate dans la pratique que la réaction de cette profession face la croissance du poids des sinistres consiste dans un premier temps ă augmenter les primes du géotechnicien : le tux actuel est voisin de $10 \%$ des honoraires encaissés. Dans un deuxième temps, l'assureur ne renouvelle pas les contrats puls ferme son portefeuille de géotechniciens. L'obtention d'une couverture d'assurance devient une préoccupation prégnante au même titre cue la recherche des affaires! C'est ainsỉ qu'un éminent confrère, à la compétence reconnue sur le plan international, n'a pas trouvêt d'assureur pour couvrir les activités de consultant privé quil voulait exercer aprés son départ en retraite de la sociẻé géotechnique qu'il dirigeail depuis plus de vingt ans.

\section{6}

\section{L'expert judiciaire}

La profession des géotechniciens est très exposée en terme de responsabilités. Les experts sort très bien placés, de part leurs missions, pour constater les dysfonctionnements d'une operation de construction dans ce domakne.

1) Au préalable, fl convient de rappeler que l'expert judiclaire, s'il doit éclairer le juge sur un problème technique, n'est pas le garant de la juste répartition des responsabilités. Il se doit de uvulgariser $n$ la technique tout en restant le plus précis possible, ce qui n'est pas un exercice simple compte tenu de la complexité crolssante des projets, notamment dans le domaine géotechnique.

Cette complexité des projets conduit l'expert judiciaire à jouer un rôle de plus en plus important dans les litiges, y compris dans l'approche des responsabilités, où souvent il lui est demandé d’indiquer des pourcentages pour chacun des intervenants à l'acte de construire. Il s'agit d'une dérive progressive qui est dangereuse et nuit à la juste répartition précitée. car elle conduit les experts techniciens à sortir de leur métier de base.
2) L'expertise connait également ses propres dysfonctionnements. Force est de constater que l'attitude des parties au cours des opérations d'expertise mérite quelques observations.

Les parties sont de moins en moins actives dans les ctossiers. Un grand nombre d'entre elles, dès lors que la garantie leur est accordée par leur assureur. se contentent de gérer le problème de franchise. ce qui conduit à une déresponsabilisation des acteurs.

Il faudrait donc que les parties s'impliquent davantage dans les litiges, notamment les géotechniciens. Ils ne font que rarement preuve d'une attitude active dans la recherche des causes, des solutions et surtout de la matrise du quantum, se contentant d'analyser le dossier clu demandeur" c'est-à-dire cuel. que part de subir au lieu d'agir. Combien de réurions dites de synthèse ne permettent pas de conclure définttivement parce qu'il manque des devis, ou que ceux-ci sont insuffisamment précis. ou qu'il n'existe pas de contre-devis, ou encore parce que certains dêfendeurs se "reveillent m et demandent des délais pour produire une solution chiffrée qu'ils auraient pu proposer des mois auparavant.

Pour obtenir une juste répartition des responsabilités, mais aussi le juste prix dun sinistre, il convient que des efforts soient faits pour fédérer l'ensemble des intervenants à l'acte de construire (maitrise d'ouvrage, lngénierie êt entreprises'), et les intéresser à la résolution des sinistres.

Les litiges faisant apparaitre phusieurs géotechniciens sont souvent l'occasion de débats u fratricices $n$, très prejudiciables à la défense de cette profession.

3) La responsabilitể des maîtres d'ouvrage est un sujet important. Chacun est conscient que, lors des régociations des marchés, la plupart des budgets sont très courts, ce qui conduil la maitrise d'ouvrage à pousser les intervenants, et notamment les gëotechniciens, à lá recherche d'économes. Ceci veut dire concrètement limiter ou modifier certaines prestations, mais aussi bien souvent faire des impasses techniques, en se disant que finalement les assureur's payeront probablement in fine.

Cette dérive doit être combattue et, pour cela, il convient d'établir une traçabilité des actions el décisions de chacun. En marché privé de bàtiment par exemple, cette traçabilité n'existe pas. Tout ce qui a été discuté en amont du projet. les modifications décidées, le programme nème du maitre d'ouvrage n'existent pas, ne sont pas contractualisés. Aujourd'hui, en dehors du cas des ventes en VEFA oủ il est possible, dans le cadre d'une expertise, de se reporter à la notice descriptive de vente pour essayer de definir quel étai le programme (ces notices sont de plus en plus succinctes également pour des problèmes de responsabilitél. l'expert ne possède que très rarement le programme envisage par le maître d'ouvrage. Il est courant d'aller expertiser un problème de dallage dans un bâtiment industriel de $15000 m^{2}$ sans qu'un cahier des charges précis n'ait été établi par le maitre d'ouvage lcharges toulantes, argeur des bandes des charıts, vitesse de ceux-ci, position exacte des racks, etc.).

Or, s'il existait un document rendu obligatoire intitule " programme du maitre d'ouvrage $n$, sur lequel pourrait d'ailleurs ètre rajouté tout ce qui a êté négocié 
et modifié tant avec. I'Tngénierte qul'aver l'Fintremrice jusqu'à la signature des marchés de travaux, l'analyse des dossiers en serait rendue plus aisée at le rôle des maîtres d'ouvrage serait bien clarifié

4) La mission de diagnostic sur existants est aussi un sujet important. Les chantiers en site urbain, comportant des travaux de réhabilitation lourds, sont de plus en plus nombreux. Or, les experts constatent que ce type de mission de diagnostic est souvent négligé, et la plupart du temps se résume à quelques sondages. Il en est de même des avoisinants où l'étude de la ZIG ‘zone d'influence géotechniquel n'est que très rarement effectuée.

On voit encore trop de mission de contrổle technique " solicité w sans que les volets a existants ) ou u avoisinants $n$, complémentaires et facultatifs, n"aient été retenus par le maitre d'ouvrage. Ces volets devraient être systérnatiquement inclus dans la mission solidité.

Le nombre de sinistres relevant de ce problème est croissant et prend une dimension particulièrement lourde Iorsque l'entreprise est au forfail, avec la plupart du temps un arrêt de chantier et des prêjudices êlevés.

5) La méthode observationnelle, dëfinie par les Eurocodes, devrait être plus largement utilisée (sutvre le comportement de l'ouvrage pour" s"assurer que celui-ci est conforme aux prévisions, définir les moyens à mettre en ceuvre et les coults si tel n'est pas le cas). Cette approche pragmaticue permettrait de tégler les problèmes techniques en cours de chantier, et non par la suite dans un cadre d'assurances.

6) La nécessité d'élargir la mission de maitrise d'ceuvre d'exécution, en y associant le géotechnicien, devient une priorité absolue, avec une présence quasi constante sur le chantier même pour les chantiers de moyenne importance (cette mission élargie est déjả pratiquée dans certains pays). Comment peuti-on encore assurer une mission de direction de chantier, clont la complexité est croissante, en se contentant de la présence hebdomadaire de " l'architecte n comme au xix" siécle ? La norme NFP 94500 prend ici toute son importance.

Cela est d'autant plus vrai que l"on assiste à une perte de technicité de l'encadrement de chantier dans les entreprises, notamment par des départs massifs d'un personnel expérimenté en retrajte lors de la dernière décennie, et que l'on compense difficilement actuellement par l'apport de jeunes cadres manquant encore d'expérience.

8) Tout le monde, sauf probablement les maitres d'ouvrage, s'accorde sur le fait que la forfaitisation du lot a fondations m devrait être proscrite. Cela supprinerait bon nombre de litiges.

9) La notion d'impropriété à destination est difficile 永 apprécier pour un expert, dans un certain nombre de cas. Limiter et préciser cette notion serait probablement sage, tant elle paraît conduire. au moins pour un technicien, à une dérive du système.

Cette dérive est particulièrement éviuente dáns ies dossiers où la pathologie est faible ct oủ, malgré tout, les solutions de réparation adoptées sont souvent lourdes, en vertu du principe de précaution.

Les experts qui pratiquent de façon trop systéma- tirgte ce principe revraient prendre conscience gu'ils agissent dans le sens d'un appauvrissement du débat technique et donc de leur rôle.

10) Enfin, la gèotechnique est une matière infiniment complexe. Cest pourtant l'un des domaines où l'on rencontre le plus d"experts généralistes.

\section{7}

\section{L'avocat et le juge}

Dans la tradition et encore à ce jour, les bureaux d'études géotechniques sont des entités restreintes. parfois limitées à quelques hommes, dépourvues de service juridique et qui ne savent pas préserver leurs droits dans le cadre des relations contractuelles gui les animent.

C'est le plus souvent démuni et face å un flou artistique que l'avocat devra défendre de tels acteurs de la vie économicjue, et dans un domaine particulièrement rigoureux pat" sor contexte financier et sa législation.

Avant l'édiction de la norme NF P 94-500 en juin 2000, les contrats, qui se résumaient le plus solvent à une énumération de sondages aux noms incompréhensibles pour tout sujet extérieur à ce monde restreint de la géotechnique, ne donnalent aucun élément de nature à permettre à l'avocat de défendre le bureau d'études et éviter sa condamnation, le plus souvent exclusive, dans le cadre de sinistres relatifs au sol.

L'avocat des géotechniciens conserve une position fragile devant les juges et cela est aggravé par fe talent des confrères intervenant pour d'autres constructeurs mieux organisés et dont la science est plus certaine. "I est aisé d’interpréter à l'infun les limites d'une mission géotechnigue pour la faire juger insulfisante eq faire imputer ce manquement au bureau d’études concerné.

En effet, en application d'ume jurisprudence constante, les juges se montrent sévères à l'égard des professionnels et en particulier des spécialistes en géotechnique puisqu’ils sont bes seuls à appréhender leur art sans pourtant le maîtriser du fait des aléas de la nature.

Le géotechnicien est souvent percu à tort comme le maitre d'cevvre des infrastructures. Les limites de ses prestations sont difficiles a definir strictement. Il apparaît alors comme le garant du a bon sol net le risque du sol est reporté abusivenent sur cet intervenant à l'acte de construire, alors qu'il intervient le plus souvent en amont et r'est pas associé à l'élaboration ou la construction du projet.

Les juges, mal informés, rarement spécialisés en droit de la construction, sont enclins à effectuer un amalgame en considérant que le détenteur de la science géotechnique particulièrement spécificue est le responsable des dommages résultant du sol. C'est pourtant souvent un choix économique ou constructif auquel le géotechnicien n'a pas participé qui conduit ả la survenance de désordres.

La norme sur les missions d'ingénjerie géotechnique a donc une fonction d'enseignement des juges qui dovent dire le divit nus sublemert au regard des dispositions strictes du contrat mais également de la norme.

Maís les responsabilités sont appréciées en fonction d'une notion plus large, les règles de l'art. Les nommes 
techniques ne roprócsntent pas touiours les règles de l'art, données actuelies de la science. En vertu du décret du 26 janvier 1984, la norme est uniquement une donnée de référence. D'ailleurs, le respect de la norme ne permet pas d'exonérer de la responsabilité fondée sur une obligation de résultat : dans le cadre du régime de la responsabilité de plein droit de l'article 1792 du code civil, le géotechnicien a peu de chance d'échapper à une condamnation in solidum lorsque le dommage relève du sol, donc nécessairement de sa sphêre d"intervention.

En conséquence, le géotechnicien doit impérativement remplir son devoir de conseil en informant des aléas inéluctables et des limites de sa mission pour voir les autres constructeurs ou le maître de l'ouvrage assımer un risçue pris délibérément.

Dans ces conditions, la défense est aisée et la sanction du juge sera applíquée à ceux qui sont réellement responsables.

4.8

\section{Les conditions d'intervention du géotechnicien}

La loi a été créée en priorité pour la protection des maîtres d'ouvrage " individuels » qui sont effectivement incompétents par nature mais également peu enclins ă dépenser pour les fondations de leur pavilor. Hormis les cas de sécheresse - dont il semble qu'elle soit de plus en plus routinière ! - l'expérience suffit généralement au géotechnicien local et expérimenté pour concevoir un projet de fondations adapté, sur la base d'une reconnaissance très limitée quí ne sert qu'ã vérifier que le terrain est bien identique à celui du voisin pour lequel il est intervenu l'année précédente.

En revanche, nombre de sinistres conséquents concernent des ouvrages collectifs ou publics dont les maitres d'ouvrage sont techriquentent compétents et se sont immiscés dans le processus de construction en amont de la signature des contrats, dès lors qu'ils ont imposé tout ou partie du programme de ieconnaissances au géotechnicien qui est forcé d'en accepter les conditions sous peine d'aller chercher ailleurs de quoi exercer ses talents. Cette inmixtion disparaît naturellement dans le dossier qui sera eventuellement plus tard entre les mains de lexpert judiciaire.

Que dire des pressions subies par le géotechnicien pour édulcorer les réserves qui l'amèneraient à déconseiller des fondations superficielles au profit de fondations profandes, ces dermières clisparaissant au fil des révisions du rapport lorsque se précisent les justifications de l'ouvage? On connait ainsi le cas, extrêne il est vrai, d'une grande surface qui a subi un tassement différentiel de plus d'un mètre à cause d'un choix de fondation inapproprié mais finatement accepté par l'ensemble des intervenants, sous la pression, ron consignée, du maître d'ouvrage.

Que dire du caractère forfaitaire des contrats relevant de la géotechnique, tant au stade des études imissions d'ingénierie et exécution des investigations géotechniques) qu'au stade des travaux (notaminent fondations, améliorations de sols, soutènements) ? Le forfait est le mode de remunération qui fait supporter au contractant le maximum d'aléas. En conséculence, il n'est à utiliser que lorsque le contexte du projet est bien défini, avec des risques résiduels clairement identifiés : cela ne peut être le cas qu'er fin d'etape de conception!
Ansi, demander de garantir par avance gun ouvrage complexe et dans un site difficile, dont on re connaît ren ou peu au départ, se comportera conformëment aux prévisions et ce, clans le cadre d'ur forfait, est à peu près équivalent au fait de demander au chirurgien du cerveau de vous assurer de la réussite de l'ablation d'une tumeur, dont il n'a encore aucune caractéristique, dans le cadre d"un forfait financier limité et dans un délaj de guérison estimé à la dernijoumée près.

\section{5}

\section{La pratique géotechnique}

Le numéro 32 de la Letre de la géotechnigue (septembre 2003 leproduisait in extenso le texte d'une conférence donnée le 29 mars 2003, a Youndé. par l'IGPC Jean-Pierre Magnai sur uLes normes et la pratique de la géotechnique

La figure présentée en annexe 3 , extratte de ce document, illustre clatirement l'emprise croissante du donaine nomatif sur l'exercice de la profession.

\section{L'auteur y apportait les commentaires suivants}

"De fait, la nome jowe deux rôles : celui d"une référence technigue el celui d un document contractuel.

"En tant que référence technique. la nome peut limiter la responsabilité personnelle de l'exécutant d'un essai ou d'une étude. Dans certains pays (comme l'ancienne Union Soviétiquel, l'application de la nome faisait d'allewrs totalement disparaftre cette responsabilité. Mais dans la majorité des pays, le projeteur garde ta responsabilité penale de ses projets, qu il ait ou non appliqué des normes, ce químpose qu'il conserve une certaine liberté par rapport à la lettre des nomes. Pour que les normes jouent pleinement le rôle de référence technique, id faut d'alleurs qu'elles couvent l'ansemble des processus qui contribuent à l'activité des ingénieurs géotechniciens. Cela n'est pas actuellement le cas en France, où des textes réglementares coexistent avec des normes et avec des textes sans statut juridique, et l'établissement d'wne collection de textes courrant l'ensemble du domaine de la géotechnique semble pratiquenent impossible, fute de temps et dargent pou' réaliser ce travail mais aussi parce que l'on mangue de spécialistes disponibles et possédant les comaissances et l'expérience núcessaires.

"En tant que reférence contractuelle, la rorme facilite l'expression de la conmande d'une étude géotechnique ou de l'exécution des travaux. Mais elle ne dispense pas le client de décider luj-mene la nature et les caractéristiques de l'ouvage qu"il désire. Maìres d'ouvrages et maitres d'ceure doivent aussi rester conscients que la référence à une nome peut suffire à définir les spécifications techniques d'une prestation mais pas les quantités correspondantes et que les nornes n'empëchent pas l'existence d'offres anomales. Ils dowent non seulement définir les quantités mais aussi les spécifications que le texte de la norme peut leur demander lce qui suppose que lon connaisse les nomes awxquelles on fait référence !). Entin. il peut être utile de verrifier que les normes (et autres spécifications techniques) sont rellement appliquées, ce que les systèmes de qualification, certification ou accéditation ne garantissent pas de façon certaine, $y$ 


\section{La norme NF P94-500 sur les missions d'ingénierie géotechnique}

La nome NF P94-500 sur la a classification et Jes spécifications des missions d'ingénierie géotechnique $n$, tout d'abord éditée en juin 2000, a fait l'objet d'une révision importante publiée en décembre 2006. Cette norme précise le contenu et les limites des mis sions d'ingénferie géotechnique ainsi que leur enchaìnement nécessaire au cours de la conception, de la réalisation et de la vie d’un aménagement de site ou d'un ouvrage pour contribuer à la maitrise des risques liés aux aléas géologigues. Elle précise également le contenu et les limites des prestations dinvestigations géotechniques qu'ill y a lieu d'effectuer pour la bonne exécution de chaque mission d’ingénierie géotechniçue. Ĺextrait de la nome a 4 . Classiftcation et enchaînement des missions types d'ingénierie géotechnique », reporté en annexe 4 , donne une description synthetique des missions à réaliser successivement à chacune des étapes d'études et de réalisation d'un profet.

\section{- Étape 1: études géotechniques préalables}

L’étude géotechnique préliminaire de site permet de définir un modèle géologique préliminaire et certains principes généraux d'adaptation d'un projet au site, avec une première identification des risques géologiques qui sont inhèrents au site.

L'étude géotechnique d'avant-projet permet de définir les hypothèses géotechniques à prendre en compte pour l'établissement de l'avant-projet des ouvrages géotechniques à l’étude, certains principes généraux de construction envisageables avec une éventuelle ébauche dimensionnelle de quelques structures types.

\section{- Étape 2 : étude géotechrique de projet}

Létude géotechnique de projet permet, en phase "projet $n$, de finaliser les hypothèses géotechniques à retenir pour le dimensionnement au niveau projet des ouvrages géotechniques. Elle fournit les notes techniques sur les méthodes d'exécution proposées et les valeurs seuils associées pour l’application du "dimeri sionnement interactif $n$, les notes de calculs de dimen. sionnement au niveau projet, l'approche des quantités, des délais de réalisation et des coûts. En phase u assis. tance aux contrats de travaux n, elle foumit les documents de consultation des entreprises specialisées, une liste des entreprises compétentes pour les travaux à réaliser, et l'analyse technique de leurs oftres.

\section{- Étape 3: exécution des ouvrages géotechniques}

L'étude et le suivi géotechniques d'exécution permettent, en phase a étude $\%$, de valider les hypothéses géotechniques retenues pour le dimensionnement au niveau exécution des ouvrages géotechniques, fourrissent les méthodes et conditions de leur exécution et définissent les mesures d'adaptation ou d'optimisation et leurs conditions de mises en ouvre en fonction des observations (relevés ef auscultations) faites en cours de chantier par le suivi géotechnique qui lui est indissociable. La phase " suivi b de l'exécution permet de valider les donnëes géotechniques retenues pour les études d'exécution à partir des observations faites en contiru sur le chantier, do déclancher à temps si nécessaire les adaptations et les dispositions constructhes prédéfinies, de foumill les éléments nécessaires gour l'établissement du dossier de fin de travaux et des tecommandations de maintenance.

La supervision géotechnique d'exécution permet, en phase "supervision de l'étude d'exécution $n$, de valider l'étude géotechnique d'exécution, et en particulier le programme d'auscultation et de valeurs seulis associées ainsı que les adaptations ou optimisations potentielles proposées par les entreprises en fonction des observations faites en cours de chantier. La phase "supervision du suivi d'exécution " pernet, par une intervention periodique et discontinue sur le chantier de valider pour le maitre d'ouvrage le contexte géotechnique tel qu'observé par les entreprises. le comportement observé de l'ouvrage en cours de construction et des avoisinants concernés, les adaptations ou les optimisations proposées en temps réel par les entreprises.

Parallèlement à ces missions d'ingénierie géotechnique qui sont à réaliser successivement en suivant les étapes d'élaboration et de réalisation de tout projet, la norma définit la mission de diagnostic géotechnique qui peut être entreprise pendant le déroulement d'un projet ou au cours de 1 a vie d'un ouvage. il a pour objet d'étudier, dans le cadre d'une mission ponctuelle et stricte. ment limitative, un ou plusieurs éléments géotechniques spécifiques: il intègre une enquête documentaire et la valication des données géotechnicues nécessaires au diagnostic. Si ce diagnostic géotechnique conduit à préconiser une modification de travaux en cours ou la réalsation de travaux. l'enchaînement des missions définies précédemment est alors à lancer.

Les missions des étapes 1 et 2 de conception ainsi que la rission de supervision géotechnique d'exécution de l'étape 3 sont normalement réalisées à l"initiative et pour le compte du maitre d'ouvrage. La mission d'étude et suivi géotechniques d'exécution est usuelement réalisée à l'initiative et pour le compte de l'entreprise.

Lavant-propos de la nome est consacré à la u PToblếmatique de la naîtise des risques liés aux aléas géologiques n qui est particulièrement importante pour cette discipline qui étudte le comportement des terrains en relation ou non avec des ouvrages existants ou à construire. En effet, les sols sont Fondamentalement très hêtưrogènes et présentent des discontinuités. Procẹdant par investigations géotechntques ponctuelles, l'ingénieríe géotechnique ne peut prétendre à une connaissance exhástive des sols qu'elle étudie. Quels que solent le maillage et la nature des investigations géotechniques réalisees, le sol gardera toujours une part importante d'inconnues et d'incertitudes : la géotechnique n'est pas une science exacte. Ainsi, l'enchainement des missions géotechniques défini dans la nome est une procédure rigoureuse qui permet la réduction progressive des incertitudes relatives à la nature et au comportement des sols étudiés, au fur et à mesure de l'élaboration du projet et de sa réalisation. La maîtrise des risques géologiques, dunc des coûts el des délais qui en découlent pour toute operation de construction, ne peut pas atteindre un niveáu satisfaisant si cette procedure n'est pas respecte.e. En particulier:

- le budget d"une opération, et a fortiorj un forfait de fondations ou d'ouvrages géotechniques, ne peuvent ètre établis avec un minimum de fiabilité sur la seule 
base des conclusions d'une éłude géotechnigue préliminaire de site $(\mathrm{G} 11)$ ou d'une étude gétechnique d'avant projet (G12) : l'étude géotechnique de projet (G2) est indispensable ;

- la non-réalisation par une ingénierie géotechnique des missions d'étude geotechnique de projet (G2). d'étude et suivi géotechniques d'exécution (G3), de supervision géotechnique d'exécution (G4) est souvent a l'origine de sinistres car seul l'enchainement de ces missions permet de définir les actions préventives possibles vis-ả-vis des risques liés aux aléas géologiques (incertitudes résidueltes, variabilité naturelle des paramètres géotechniques), les dispositions à mettre en cuvre pour détecter leur survenance le plus tôt possi. ble et les actions curatives pour en minimiser l'impact s'ils se réalisent.

En conclusion, l'application de cette nome sinscrit dans l'action plus générale de management de projet avec processus de maitrise des risques en toute transparence pour un partage équilibré et équitable des risques et donc des responsabilités. Elle doit se traduire en particulier par une maturité géotechnique suffisante du projet et un suivi assidu des travaux pour une vision partenariale des problèmes à résoudre gui seront détectés au plus tồt. Ainsi l'ingénierie géotechnique aura les moyens d'apporter sa contribution indispensable à la réalisation d'ouvrages de qualité.

\section{8}

\section{Les autres documents techniques de référence}

Les nombreux documents quil constituent le rêférentiel technique géotechnique illustré sur la fịure 1 sont d'origines et de natures très variées : documents techniques unifiés (DTU) nomes européennes et françaises, nomes étrangères, cahiers des charges validés par des contrôleurs techniques, fascicules édités par les instituts comme les laboratoires des ponts et chaussées, recommandations ëtablies par des organisations professionnelles. mais aussi des logiciels ou des lives rédigés pár des géotechniciens reconnus et quí font eux aussi partie du corpus commun à la profession.

Certains ouvrages, comme les fondations, sont soumis à des documents très détaillés qui sont toutefois différents selon cu'ils sont destinés aux ouvrages publics (fascicules du CCTG) ou privés (DTL).

Il est important de noter que tout document est eta bli un moment donmé, avec les connaissances techniques et le retour d'expérience disponible à ce moment là. Mêne si les rédacteurs sont conscients de ces limi tes, il peut arriver que l'utilisateur gènéralise, sans en prendre toujours conscience, les prescriptions a des situations quil n"avajent pas été envisagées au moment de la rédaction.

Les méthodes de calcul considèrent très souvent des cas tdéaux qui ne correspandent pas à la complexité des ouvrages à construire dans la pratiqué. L'expérience du géotechnicien est alors essentielle pour adapter ces méthodes, choisir les paramètres.

Ces questions ne sont d"ailleurs pas limitées à la géotechnique. Par exemple, dans le domaine du béton armé, les tiurocodes ne sont pas adaptés au calcul de la fissuration des éléments autres que rectangulaires et d'épaisseur relativement limitêe. S'il n'est pas interdit d'utiliser d'autres méthodes de calcul le concepteur est démuni forsqu'il doit les étayer par des essais en lahoratoire en vтaie grandels nu des mesures sur des ouvrages réels. On se heurte alors à des débats sans fin où la véritable question est - in fine - cel le de la responsabilité en cas de problème.

Beaucoup de procédés ne sont pas couverts par les méthodes de conception. C'est évidemment le cas des procédés nouveaux, pour lesquels les entreprises développent des cahiers des charges particuliers, généralement avec le concours de contrôleurs techniques ou de services techniques publics. Ces cahiers des charges sont établis sur une base d'essais et de letour d'expérience nècessalrement limitée.

Dans ce domaine, il est donc important que les clients publics favorisent la réalisation d'ouvrages de référence, ouvrages qui sont ensuite utilisés pour justifier une application plus large de ces techniques et procédés innovants. Les ouvrages de référence sont aussi un vecteur important pour la promotion à l'étrañger des techniques développées en France.

Les aléas liés à ces ouvrages a hors normes it sont en général largement compensés par une mobilisation forte d'expertise pour Ieur conception, leur suivi et leur contrôle.

\section{5,3}

\section{Les investigations géotechniques}

Comme indiqué dans la colonne de droite du tableau 'I de l"extrait de la norme NE P94-500 reporté en annexe 4, l'enchainement des investigations géotechniques suit celui des missions d'ingénierie géotechnique. En effet, chaque mission s'appuie sur les résultats d'un programme d'investigations géotechniques dont la définition fait partie de la mission d'ingénierie. Chaque étape d'snvestigations géotechniques, réalisées ou non par l'ingénierje géotechmique, comporte l'exécution du programme de sondages, essais et mesures en place ou en laboratoire et l'établissement du compte rendu factuel.

Le programme d'investigations géotechnigues est établi en fonction de trois éléments majeurs:

- la nature de l'ouvrage à réaliser'

le contexte geologique, hydrogéologique et géotechnique du site

- ja zone d'influence géotechnique, zone d'interaction entre le projet, le terrain et le voisinage.

Il doit préciser la nature des investigations (type de sondages et d'essais), la quantité des investigations (nombre de points de sondages et d'essais) et leur extension (périmètre à reconnaître, profondeur, espacement entre points de sondages). En particulier, les techniques de reconnaissance utilisées doivent être particuljêrement bien adaptées aux objectifs recherchés : ansi, Jes "recommandations sur la consistance des investigations géotechrigues pour la construction de bátiments m. établies par l'LSG avec le concours du CFMS, donnent un tableau de la pertinence des techniques usuelles de reconnaissance

Toute campagne d'investigations géotechniques comporte un nombre limité de sondages et essais givi ne permettont janais de lever toutes les incerti- 
tudes inhérentes à cette science naturalle. Toutafois, ces incertitudes doivent être réduites de manière économiquement acceptable, selon le contexte du site et du projet : elles devront être prises en compte dans la conception de l'ouvrage géotechnique. En particulier. les conclusions géotechnigues qui reposent sur ces investigations géotechniques ne peuvent conduire a traiter a forfait le prix des fondations sans prise de risque, compte teru d'hétérogénéités toujours possibles (naturelles ou du fait de l'hommel et des aléas d"exécution pouvant survenir lors de la découverte des terrains d'assise.

\section{4}

\section{Les avoisinants sensibles}

Lexemple de la rue Raynouard á Paris a montré à quel point les avoishants peuvent constituer un risque de prejudice majeur. Or, si les risques attachés à l'ouvrage proprement dit sont relativement identifiables, les risques liés aux avoisinants sont très difficiles à cemer. Qui plus est, les avoisinants sont hors de la propriété du maître d'ouvrage. Dans le cas d'un soutènement, le sol soutenu est chez le voisin ou les reconnaissances sont a priori impossibles bien qu'indispensables pour délecter les éventuels accidents de sol et connaître les conditions hydrauliques.

Il y a là une limitation drastique des possibilités dintervention du géotechnicien.

I] est de la responsabilité du naître d'ouwrage ce faire réaliser les études adaptées sur les mitoyens puis de choisir, en matiere de soutènement, les solutions qui apportent la meilleure sécurité pour les mitoyens, et d'en supporter le coút.

\section{5}

\section{L'intérêt du dimensionnement interactif}

Au VIte Congrés international de la mécanicue des sols et des travaux de fondetion (ICSMEE - Moscou 1973]. Ralph Peck concluait son discours d'ouverture par ce conseil adressé à route la profession : tant que nous, géotechnichens, n'avons pas été sur le termain voir comment le conducteur de pelle réalise le fruit de notre étude, nous n'avons pas rempli notre mission. C'étalit. avec trente ans d'avance sur la nome, la demande expresse de la généralisation des missions aujourd'hui préconisées dans l'étape 3 de la norme.

La conception et la réalisation d'ouvtages complexes dans un contexte de voisinage urbanisé sensible justifient la nécessité de recourír au dimensionnement interactif. Il se caractérise par:

- des études très détaillées au stade de 睹 conception: études intégrant chaque phase de travaux, la sensibilitế de la structure à construire el celle des avoisinants. les variabilités et les incertitudes affectant les donneses géotechniques du site:

- un dispositif de suivi et d'auscultations de l'ouvrage et des avoisinants en cour's de travaux pour s'assurer que leur comportement observế est dans les limites admissibles lors de la conception, ou pour permettre la mise en cuvre d'adaptations constructives avant l'apparition de désordres majeurs.
L'application du dimensionnement interactif repose sur quatre exigences :

- les limites acceptables du comportement des ouvrages concernés doivent être établies :

- Je domaine de variation possible du comportement doit être estimé, avec une forte probabilité pour cue le comportement réel reste dans les limites acceptables;

- le programme de suivi doit être établi. pour vérifier que le comportement réel des ouvages est dans les Limites acceptables;

- un programme de mesures d"urgence doit être établi avant le début des travaux. afth de pouvoir le mettre en ceuvre sans délai de réaction si le suivi des travaux révêle un comportement des ouvrages sortant des lirnites acceptables.

Le dimensionnement interactif est ainsi un élément majeur dans le processus de la maitrise des risques liés aux aléas géologiques et de voisinage, processus particulièrement important aussi bien pour les projets complexes que pour les sites au contexte géologique défavorable ou au voisinage sensible.

Le dimensionnement interactif est toutefois incompatible avec la notion juridique du forfait qui implique que l'objet de l'engagement contractuel, contrepartie du prix fixé, soit clairement et précisément défini. Or il est clair que sỉ l'ouvage à réaliser évolue de maniêre notable, si les méthodes d'exécution doivent être rotalement nodifiées, du fait et par application du dimensionnement interactif, le prix et les délais contractuels doivent évoluer parallèlement.

\section{Les pistes d'amélioration}

Le challenge à rêlever par le géotechnicien pour apponter sa contribution à l"amélionation du processus de construction est de merier une discipline expérimentale avec un cadre juridique et dentreprise très contraignant.

Pour éviter qu'une faible pathologie géotechnique re conduise un expert généraliste à faire réaliser une rêparation lourde, des amétiorations sont souhaitables et possibles à toutes les étapes de conception, réalisation et exploitation d'un ouvrage.

\section{1}

\section{La contractualisation des risques geologiques}

L'accroissement des risques dans le domaine de la construction, constaté depuis plus d'une décennie, a des origines multiples mais les plus importantes à souligner sant les suivantes:

- une mobilisation des bonnes compétences trop souvent allégée en amont par le maître d'ouvrage, par manque de temps ou recherche d'économie abusive:

- l'utilisation des terrains encore disponibles, de qualilé géotechnique souvent médiocre, avec un voisinage de plus en plus sensible:

- des ouvrages de plus an plus complaxes, avec des exigences renforcees en termes de performances et de qualité, alors que les méthodes d'exécution sont de moins en moins adaptables aux aléas et anomalies rencontrées ; 
- des intervenants de plus en plus nombreux, donc LMe chaîne des risques et des responsabilités plus complexe;

- des délais d'études et de travaux souvent trop raccourcis:

- une maintenance des ouvrages trop souvent négligée, vaire inexistante;

- des procédures judiciaires banalisées et onéreuses, conduisant souvent a des dommages immatériels sans rapport avec le coût des réparations ou de l'ouvrage à l'origine.

Devant cet accroissement des riscues, il est apparu nécessaire à tous les intervenants à l'acte de construire que le management d'un projet intègre un processus continu de gestion des risques. Seule cette demarche permet d'obtenir un ouvrage de qualité et pérenne. tout en optimisant son coût et son délai de réalisation. Le coût final de l'ouvrage, obtenu après gestion optimale des risques et opportunités intervenus en cours de chantier, doit bien être differrencié du coüt optimal recherché en début d"opération et résultant d'un niveau de risques supposé le plus bas et d'un niveau d'opportunités supposé le plus élevé.

Cette gestion des risques doit être présente à chacune des quatre étapes d"un projet : programmation, conception, passation des marchés travaux, exécution des travaux. Elle doit être transparente, dynamique et partagée pour éviter tout transfert caché de risque et obteniu ainsi leur traitement aptimum : elle doit être axée sur leur détection le plus en amont possible at sur le contrôle de l'efficacitế des parades prévues. Elle comporte les quatre volets habituels : identification, évaluation, traitement, suivi.

Le partage des risçues doit résulter d'une démar"che volontaire et non fataliste, la prise en charge de chacue risque relevant de f'intervenant qui est le mieux à même de le maîtriser compte tenu de sa competence technique et de ses engagements contractuels.

Le maître d'ouvrage doit mobiliser une équipe de concepteurs en rapport avec la complexité de son projet : il doit vérifier en particulier que leurs compétences, les délais et rémunérations qu'il leur accorde ne sont pas de nature à accroitre le risque d'une maturitè insuffisante de son projet. Etant le béréficlaire de la construction, il doit assumer les risques inhërents à l'opération qui auront été identifiés et traités selon les règles de l'art.t.

L’ngérierte doit veiller ce que la maturité des études soit suffisante à chaque étape de la conception puis de l'exécution pour que la gestion des risques solt pertinente tout au long du déroulement du projet : c'est â ce niveau qu'elle doit assumer son devolr de conseil envers le maitre d'ouvtage. En particulier. l'ingénierie doit conseiller au maître d'ouvrage le type de contrats de travaux le mieux adapté au niveau de définition de l'ouvrage lors de la consultation des entrepreneurs. permettant le partage des risques le plus efficace et le mode de rémunération le plus adéquat.

L'entrepreneur doil valider et compléter lors de son offe les quatre phases de la gestion des risques faite en amont, compte tenu des methodes d'exécution qu'i] prévoit de metre en ouvre. Il pourra alors assumer en toute connaissance de cause les risques liés à ses propres méthodes d'exéculion, compte tenu des données du projet et des riscues identifiès.
Catc répurtition ćquilibróc des cngagements de chacue acteur dans son domaine de compétence et dans sa sphère d'influence permet une vision partenariale des problèmes à résoudre, au-delä des divergences d'intêrêt propres à chacun.

C'est dans cet état d'esprit que lá réviston de la norme NF P94-500 a été établie en décembre 2006. Le géotechnicien doit être associé aux autres ingénieries, à toutes les étapes successives de conception at de réalisation d'un projet, afin de contribuer à une gestion efficace des risques géologiques et ainsi fiabiliser le délai d'exécution. le colti à teminaison et láa qualité des ouvrages géotechniques que comporte te projet.

Les conditions générales des missions d'ingénierie gètechnique (annexe 5), établies par l'Union syndicale géotechnique (USG), rappellent au maître d'olvrage et à son maitre d'ceuvre les spécificités de ces missions et la mécessité de leur enchaînemant.

\section{0}

\section{Le contexte des contrats}

Depuis une décentale et sutout depuis la parution de la norme NF P 94-500, la pratique de la géotechnique est en constante évolution, rnais elle demeure encore solvent inadéquate. En principe, les missions d'avant-projet (G1) ne permettent pas de « forfaitiser n les fondations: en pratique, 音 ce jour, la majeure partie des opérations ne bénéficient que d"une telle étude prealable et font l'objet d'un marché forfaitaire. Ainsi, de nombrewx litiges sont relatifs à des travaux supplémentaires résultant de la variabilité de la nature du sol découverte en cours de chantier, différente de celle décrite par le géotechnicien dans son raport géotechnique préalable ou différente de celle prise en compte par l'entreprise dans l'évaluation de son forfait. Il s'agit d'un contentieux florissant dans un marché concurrenliel redoutable qui conduit les entreprises à remetre des offres sans prévoir les aléas inéluctables et souvent previsibles en matière de sol.

Pourtant le drcit peut être source de déconvenues pour de tels entrepreneurs trop optimistes.

Dans le caclre d'un marchè à forfait. en droit privé comme en droit public, l'entreprise a la charge du risque du sol à l'égarcl du maitre de l'ouvrage.

Les juridictions judiciaires se montrent séveres par application de larticle 1793 du code civil pour la construction selon un plan arrêté et convenu d"un bâtiment et admettent de maniêre restrictive le clroit à paiement des trava ux supplémentaires. Ainsi, les réserves émises aux termes du rapport d'étude géotechnique préalable doivent êre prises en compte par fentweprise [Cass. civ 3 e 21 juin 2000].

De même, le juge administratif est d'autant plus soucleux du respect du contrat conclu, dans un contexte rigoureux d'appel à la concurrence où le prix proposé constitue un élément non négligeable du choix du cocontractant. Tout le jeu serait nécessairement faussé sf on admettait une révision du prix a la hausse en fonction du coût effectif des travaux. Les sujétions techniques imprévues ne sont admises par le juge administratif que si elles ont présenté un caractère d'anomale gravité el étaient imprévisibles; les difficultés susceptibles d'entrainer l'application d'une telle théorie tiennent 
évidemment à des sujêtions liées zu sous-sol par nature imprévisible. Pour un marché forfaitaire, les sujétions imprévisibles ne peuvent donner lieu à indemnisation que si elles ont provoqué un Louleversement de l'économie du contrat [CE 6 mai 1936, Ministre de la Guerre c/ Régnier"].

Ce panorama juridique devrait conduire à développer des cadres contractuels mieux adaptés pour une appréhension nornale des risques. Dailleurs. I1 ne s'agit pas seulement d"appréciation du coût de louvrage définitif, mais ëgalement des risques engendrés dans le tenps. La vie de l'ouvrage suppose une mantenance, voire un suivi détałlé avec mise en place d'une instrumentation, permettant de pallier les effets ineluctables du temps qui engendrent des risques pouvant s'averer tragiques au plan humain. Mais n'est-ce pas ur vou pieux pour la plupart des ouvrages?

Des solutions juridiques existent ; elles permettent un partage des risques et surtoul prévoient un financement a ce titre. Solutions acquises pour des ouvrages exceptionnels, elles ne demandent qu'à prospérer, mais nécessitent une évolution des mours en France et une certaine ouverture à des praticues communautaires quí ont fait leurs preuves.

D'influence étrangère et communataire (Livre vert sur les partenariats public-privé et le droit communautaire des marclyés publics et des concessions du 30 avril 2004), le contrat de partenariat dit \& PPP y est issu de l'ondonnance $n^{\circ} 2004-559$ du 17 juin 2004 : une personne privée est chargée, par une personne publique, de financer, construire et exploiter un équipement ou un service public. Ce contrat comble un vide entre les marchés publics qui interdisent le paiement différé et les délégations dans lesquelles la rémunération se fait sur l'usager. Avec une phase novatrice de dialogue pour la passation du marché. il permet une répartition des risques en fonction des capacités de chacun des cocontractants à les assumer, le cocontractant prive pouvant prendre en charge le risque de construction et de maintenance. Thais non celui de la réglementation (mise aux nomes).

En toute hypothese, la mațise du cout' d'un projet passe par une contractualisation aclaptée à chaque étape. En particulier, dans la contractualisation avec chaque intervenank, les dêlais de remise des offes. de mise au polnt du contrat et de préparation des travaux ne doivent pas être réduits au strict minimum: il faut être conscient qu'une partie de la réussite de la prestation réside dans la qualité de sa préparation par les deux parties.

Ainsi, en amont, le maitre d'ouvage cloit préciser son programme, avec selon ses compétences techniques internes, l'aide d"un assistant à maìtre d'ouvrage et surtout de son maitre d'cuvre dès qu'il l'a déjà choisi.

La compétence du géotechnicien s'exerce au départ dans un contexte de fortes incertitudes, Ja compréhension du contexte géologique étant indispensable pour les gerer. Il doft donc être mis en place avec le maitre d'ouvrage, dès les études géotechniques préalables (étape 1 de la norme des missions d'ingénierie géotechniques). un processus de management des risques qui suscıte une motivation convergente des intervenants.

Les mécanismes contractuels permettant plus de souplesse doivent être retenus, qui donneront au géotechnicien compétent les moyens de participer à la maîrise du projet : délais d’êtudes suffisuntes modes de rênunération adaptés aux évolutions du projet et à son optimisation permanente. Ce sont les faits générateurs de coûts et non le coût lui-mêne que le géotechnicien doit aider à maitriser.

La fomule cle passation des marchés de travaux géotechniques doit être choisie sur la base de trois critères:

- le niveau de définition de l'ouvrage géotechnique au lancement de la procédure:

- le mode de partage des risques le plus efficace et transperent:

- le mode de rémunération le plus adéquat.

Le contrat sur prix mixtes forfaitaires et unitaires est celui crui est a priori le mieux adapté pour ce type de travalix oì les aléas géologiques sont toujours plus ou moins présents. Il doit être établi en respectant les fondameratalux suivants:

- une estimation s honnête n et transparente des cuan. tites pour que l'entreprise puisse comprendre comnent elles ont été calculées, un coefficient d'incitation étant introduit pour la réduction des quantités :

- la définition d'un mode de règlement des écarts pré. visibles évalués dans l'analyse de riscues ;

- la décomposition des prix unitaires et/ou forfaitaires pour permettre le cas échéant la prise en compte des problèmes susceptibles d'être rencontés et notamment des adaptations nomales en fonction de la variabilité constatée des caractéristiques des terrains.

\section{3}

\section{L'exercice de la profession}

Le texte de J.-P. Magnan cité plus haut. concluait :

a Le développenent de la normalisation est une tendarce forte de l'évolution des sociétés modemes, mème si quelques ralentendus ont pu exister entre les tenants de la normalisation et les spécialistes de la géotechnique (nomaliser les essais sur les sols ou les rocies, ou encore les calculs ou les travaux est différent de nomaliser des prises électriques. En géotechnique, la génération drexperts actuelle a crée les textes de réfêrence et les nomes... les générations suivantes verront ce qu'elles doivent deverir. Mais il parât souhaitable de respecter les particularités de la géotechnique dans cette codification des pratiques, notanment le caractère artisanal des études géotechniques (tous les cas sont unigues). II faut aussi organiser les cantriestes des spécialistes qui seront les experts de demain. „

Cette conclusion exprine, en termes diplomatiques, qu'un cadre est nécessaire à l'exercice de la profession mais que l'encadrement juridique ne crée pas lá compétence. De plus, un carcan trop fort ou inadapté risque d'enlever à l'homme de l'art toute possibilité de mettre a profit son expérience pour prendre des risques a raisonnables zace aux situations non couvertes par la réglementation en viguevi.

Elle ouvre ainsi tes pistes envisageables poul" amélioret l'exercice de la profession de géotechnicien face aux partenaijes contractuels :

- intégrer la culture de la norme dans la formation scolaire, universitaire et professionnelle des spécialistes afin qu'ils en connaissent le contenu technique et les implications contractuelles; 
- éliminer de la norme toute orescrintion imcomna tible avec cet incontoumable duo de situations que constituent l'inapplicabilité de la statistique classique au matériau « sol nassociée au caractère a prototype a de la plupart des ouvrages dès lors qu"il ne s'agit pas du néme pavillon d"un lotissement en rase-campagne.

\section{4}

\section{L'accompagnement du géotechnicien}

Le géotechnicien est J'intervenant le mieux qualifié pour aider le maître d'ouvrage à mattriser les risques liés aux aléas géologicues, l'aléa gèologicule étant défini comme étant un évènement géologique non prévisible dans l'espace et dans le temps concernant les caractếristiques d'uti site.

\section{Les risques géologiques sont tributaires:}

- du contexte géotechnique du site. Sa madélisation est toujours simplificatrice et réductrice, alors que le problème rencontré est souvent liê a généité locale dont les conséquences peuvent ếtre amplifiées par une détection tardive. Cette spécificité justifie la réalisation des études et investigations géotechriques par étapes successives pendant la phase de conception, puis le suivi géotechnique pendant la phase de travaux, afin d'obtenir une meilleure maitrise des risques liés aux incertitudes restantes, aux variabilitếs des paramètres et aux aléas géologiques;

- des spécificités de l’ouvrage à construire, et en particulier de la sensibilité de sá structure vis-á-vis des jncertitudes et variabilitếs sur les paramètres gêotechniques

de la zone d’influence géotechnique (ZIG), volume de terrain au sein duquel il y a interaction entre le projet (đu fait de sa féalisation et de son exploitation) et l'environnement (sol et ouvrages avoisinants). La maîtrise de l'impact sur l'environnement passe part une bonne connaissance du contexte géotechnique au droit de la ZIG ainsi que des ouvrages avoisinants (structure et conditions de fondationl.

Ainsi, l'enchainement des missions d'ingénie. rie géotechnique en accord avec le déroulement du projet (conception, travaux, maintenance) conduit le géotechnicien à avoir une vue plus globale sur la bonje aclaptation du projet áu contexte géotechnique du site en particulier, le suivi géotechnique Iors des travax permet de détecter à temps les anomaties par rapport aux prêvisions et de metre en oevwre si nécessaire Jes adaptations constructives.

L'accompagnement du géotechnicien contribue à une meilleure maitrise des risques géologiques :

- au stade de la conception, par une évaluation des incertitudes et des variabilités des paramètres géotechniques influents, par une connaissance de la sensibilité de l'urvrage projeté et de l'environnenent, par la pré. vision des dispositions constructives à mettre en aeuvre eventuellement, par une identification des risques inhérents à budgéter, par la conception d"un dispositif de contrôle et de surveillance de l'environnement et de l'ouvrage projetéf pour valider la concordance entre prévision et réalité
- nonriant lexérultion dec travaly par la mise en neivre et le suivi du dispositif de contrôle et de surveillance avec définition de la valeur des seuils de vigilance et dªlerte, par la rêlisation à temps des dispositions constructives prévues à l'avance si nécessaire ;

- après les travaux, par une mantenance adaptée au contexte géotechnique at à l'ouvrage.

\section{$6=$}

\section{Le partenariat des assureurs}

Pour que les assureurs puissent remplir leur rôle, il faut rétablir entre les constructeurs et eux une relation de confiance fondée sur le partenariat. Cela implique un processus continu de la gestion des risques mobilisant tous les intervenats y compris les assureurs qui pourront alors plus facilement mettre en place une couverture des risques résiduels aléatoirés concernant aussi bien les dommages à l'ouvrage qu'aux tiers.

Instaurée pour tout projet, cette gestion des risques, uransparente, partagée et dynamique, doit permettre à chaque constructeur et en particulier au géotechnicien d'optimiser son programme d'assumances amuleles pour solt activité propre, aussi bien pour couvrir sa responsabilité civile professionmelle classique que sa responsabilité a décennale bâtiment i qu'il convient: de mieux encacrée.

Pour ce qui concerne le géotechnicien, le marché de l'assurance pourra alors lui proposer des conditions de couverture de ses responsabilités plus en adèuation avec son poids économique : en limitant raisonnablement sa couverture d'assurance entre un et trois millions d'euros par sinistre selon sa taille, le géotechnicien retrouvera un assureur et des cotisationg raisonnables.

Pour ce qui conceme le matre d'ouvrage, selon la complexité et le coût de son projet, il pourrá alor's mettre en place une u complémentaine de groupe r pour couvrir la plupart de ses risques au-dela de la couver ture apportée par chaque constructeur: en effet, mobilisé plus en amont, son assureur pourra juger de la qualité de la gestion dynamiçue et transparente des risques et metre ainsi en place un programme optimal et cohérent.

Cette démarche globale de gestion des risques permettra une juste répartition des risques et responsabilités, condition indispensable pour qu"un programone d’assurances optimuth et cohérent pulisse être mis en place pour la couverture de risques mieux appréherdés, et cela quel que soit le coùt global du projet, ce qui n'est plus le cas aujound'hui.

\section{6}

\section{La pratique des experts}

S'agissant de la pratique expertale dans la gestion des sinistres, et reprenant les propositions faites au Chavilie 4.6, un retiendla comme vistes d"amélioration possibles

- que les parties s'implicuent davantage dans les litiges, notamment les gétechniciens. L'expertise est la seule situation où le géotectnicien dispose, en prin- 
cipe, de toutes les picces d’un dossior dont it n'a eu jusqu'alors que ies bribes qu'on a bien voulu lui transmettre. A lui d'en profiter:

- aux ingénieurs qui le consuitajent, Albert Caquot donnait toujours ce conseil reçu, jamais démenti par l"expérence : "Un ingenieur est d'abord un "dessinateu" et plutôl que de "poser" une question, "dessinez-la". Vous verrez apparaître spontanément l'essentiel de la réponse que vous attendez. D Dans le cadre d'une expertise. le dessin ou le plan bien fait a, de plus, la vertu de ne pas pouvoir faire l'objet d'interprétation ejronée, voire malicieuse. On ne peut qu'encourager les experts et les conseillers techniques des parties a recourir systématiquement aux documents dessinés comme support écrit et oral de l'expertise. L'expérience prouve que les juristes savent être aussi sensibles à cette approche "bande dessinee y des dossiers exposés;

- pour obtenir une juste répartition des responsabilités, mais aussi le juste prix d'un sinistre, il convient que des efforts soient faits pour fédérer l'ensemble des interverants à l'acte de construire (maîtrise d'ouvage, ingénterie et entreprises), et les intêresser à la résolution des sinistres

- la dérive de la pression des maîtres d'ouvrages sur la consistance des études géotechmiques doit être combattue et pour cela il convient d'établir une traçabilitê des actions et décisions de chacun en rendant obligatoire un document intitulé " programme du maitre d'ouvrage $n$, sur lequel pourrait d'ailleurs être rajoutế tout ce qui a été négocié et modifié tant avec l’ingénierie qu'avec l'entreprise jusqu'à la signature des marchés de travaux :

- les volets a existants n ou th avoisinants devmaient ëtre systénatiquement inclus dans la mission solicité du contrôle technique. Le propre du domaine des soutènements et - par extension - du donaine de la stabilité des pentes, est que la ZIG (zone d'influence géotechniquey. c'est-à-dire le volume des sols concernés par les équilibres mécaniques et les amplitudes dé déplacements à respecter, se sique la plupart du temps hors de l'emprise du projet. Les reconnaissances y sont souvent difficiles, voire impossibies - techniquement ou de par l'obstruction des propriétaires voisins - et les risques associés peuvent avoir des consequences sans commune meșre avec le coût du projet :

- le géotechnicien doit être assocłé à la mission de mầbrise d'ceuvre d'exécution en application de l'étape 3 de la rome géotechrigue

- préciser la notion d’impropriété à destination et en. limiter l'étendue de façon à favoriser les solutions réparatoires « raisonnables »

- porter une attention particuliêre à l'zgrément des experts spécialistes en géotechnique ế. surtout, à l'adéqualion de leur désignalion lonsque l'expertise relève de leur compétence. A défaut d’une refonte des listes existantes, mettre en place des listes de sapiteurs spécialistes, agréés par la profession selon un mode d'élection à définir.

\section{7}

\section{Conclusion}

La recherche du a risque zero m, le développement de la normalisation, la désignation d'un responsable à tout préjudice sont des tendances fortes de notre société en ce debut de xxt" sic̀le. tendarices qui s"intjosent évidemment à la pratique de la géotechnique.

Or la géotechnique est un domaine spécifique qui doit tenir compte d'un acteur d'humeur variable. difficile à appréhender êt généralement insensible aux règles et normes édictées par les homrnes : le sol.

Oulre le sol, ce document a passé en revue les nombreux acteuss d'un projet de construction : majtre d'ouvrage, maître d'ceuvre, entreprise, assureur. contrôleur technique, expert judiciaire, avocat, juge. sans oublier le géotechnicien. Leurs logiques propres. leur rỏle dans les projets, leurs relations contractuelles, leurs responsabilités, notam-tient vis-à-vis d'un encadrement juridiçue complexe et pas toujours adapté à la pratique géotechnique, ont étể analysés.

A l'issue de cette analyse des effets de lencadrement juridigue sur la pratique géotechnique, les principales. pistes d'amélioration, issues de la rêalité gếotechnique pour ure pratigue juridique rénovée, sont les suivantes.

Metre en place w. veritable managentent des risgues à chacune des étapes des projets : programmation, conception. passation des marchés de travaux construction. Transparente, dynamique et partagée, cette gestion des risques doit éviter" tout transfert caché de "responsabilité et assurer une traçabilité des décisions et des évolutions du projet.

Favoriser l'implication plus continue du géotechnicien au cours du projet. Les interventions trop souvent fractionnées en lots indépendants ne permettent pas au géotechnicien d'accompagnen le projet pas une analyse cohérente et globale des risques.

mpliquer plus étroitement les assureurs dans la gestion des risques. afin de développer des programmes d'assurance cohérents et représentatifs du poids économique et des responsabilités réelles des différents acteurs du projet. Favoriser la rémuneration directe par l'assureur du contrôleur technique, homme de l’art de l'assureur sur le terrain et cul est pourtant choisi par le maitre d'ouvage après mise en concurrence.

Recourir plus fréquemment aux contrats sur prix mixtes forfaitaires et unitaires et développer les contrats en partenariat. Couramment utilisés dans certains pays européens, ce type de contrat fournit un environmement contractuel permettant de gerer les risques et d'optimiser le projet dans une relation gagnant/gaglant entre tous les intervenants. La forfaitisation des contrats provoque de nombreuses situations conflictuelles lorsque le sol est susceptible de modifier significativement la prestation à réaliser. La notion de forfait n'est certainement pas adaptée au dimensionnement interactif des ouvrages géotechniques.

Former et sensibiliser les juristes et jes ingénieurs aux spécificités de la gétechniqué : claque ouvrage ou presque est unique, le sol est un matériau hétérogène et évolutif qui répond mal au formalisme statistique développé pour les matériaux industriels, comme le béton armé. Si ura cadre normatif est nécessaire la norme ne peut pas couvrir toutes les situations rencontrées dans la pratique. La norme doit laisser la possibilité aux hommes de l'art d'adapter les réponses et les techniques aux réalités du terrain. Elle doit aussì laisser aux entreprises la possibilité d'innover avec des solutions techniques, validées sur des arojets de référence en France et destinées à renforcer la compétitivité de hos entreprises à l'étranger. 
En cas de litige, faire intervenir des evperts specialistes en géotechnique et intéresser effectivement les parties à la résolution des conflits an plus vite et au moindre coltt. L'expertise judiciaire est wne situation où la mise à disposition de l'ensemble des pièces d'un dossier permet aux parties une approche globale de l'enchałnement des décisions et de la définition des responsabilités des acteurs.

\section{Bibliographie}

Allagnat D. (sous la dir. de) - La methode absemationnelle pour le dimgraionmement interactif des ourrages, Presses des Ponts et Chaussées, Paris, 2005.

Carriere M.-L. - Les obligations des buteawa détudes de sols, rapport pour l'USG, juin 1996.

Cartière M.-1. - La responsabilité en matiére de sol. Collaque Justice of Guntuction. Faris, 2004

Caston A. - La responsabilité des consitucteurs, éditions Le Monitelir. Paris, 1995

Catz J. - Les constructerus we lisgue du sol.

Colkoque du 18 septembre 2006 au Palais du Luxembourg a Ris. ques, Responsabilites. Assurances m, organisẻ par Syntec Irné.

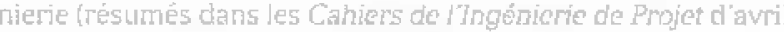
2007 et textes sur le site www.synter-ingenierte.fr].

Karila J.-P. - Resporsalyilte assurance construction : la reforme du 8 Jun 2005. Le Monitew", 16 septembre 2005.

Joumées d'Etude du GIS MA-GenCi du 23 mars 2006. Actes de la journée dans les Anndes du bätinent et des travaux publics. aoủt êt septemlore 2006

Magnan J.-P. - Les nomes et la pratiqua de ta géotechrigue. Conférence donnée à Yaoundé le 29 mars 2003. Lá Letro de la Géotechnique $n^{\circ} 32$ (www geotechnique.org ) 2003

Malinvaud P. - La responsabilité en matière de construction aprẻs

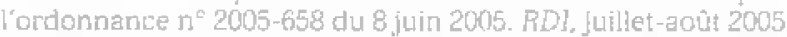


cation et spectications (AFNOR, novembre 2006)

Rapport sur l'Assurance Construction de l'inspection générale des finances et du conseil géneral des Ponts et Chaussées octobre 2006].

Syntec Ingénierte - Liwre blanc Responsabilité at assumances. Syn tec Ingenterje, mars 2005

USG - Recommandations sur la consistance des investigations géotechniques pour la construction de bâtiments. USG, janvier 2005 modifié mars 200 r.

USG - Colloque du 20 mai 2005 au Palais du Luxembourg a Ris. que Construction : le cout du sol m, or dantisế par IUSC.

\section{Annexes}

\section{A.1}

\section{Article 1134}

« Les conventions légalement formées tiennent lieu de loi ca ceux quil les ont faites. is

« Elles ne peuvent être revoquées que de leur consentement mutuel, ou pour les causes que la loi autorise. 2

"Elles doivent être exéculèes de bonne fol. n

\section{Article 1135}

« Les conventons obligent non seulement à ce qui y est exprimé, mais encore a toutes les suites que l'équitế, l'usage ou la loi donnent ả l'obligation d'après sa nature. b

\section{Article 1147}

" Le débiteur est condamné, s'il y a lieu, au payement de dommages et intérêts, soit à rajson de l"inexếcution de l'obligation, suil à l'atson du retard dans

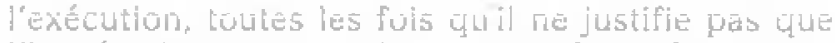
linexécution provient dune cause étrangère qui ne peut lui être imputée, encore quil n'y ait aucune mauvaise foi de sa part.

\section{A.}

\section{Loi Spinetta du 04 janvier 1978 (extraits)}

Art. 1. Lanticle 1792 du code civil est remplacé par les dispositions suivantes :

Art. 1792. Tout constructeur d'un ouvtage est respon sable de pleiı droit, envers le maître ou l'acquéreur de l'ouvage, des dommages, mểme résultant d'un vice du sol, qui compromettent la solidité de l'ouvrage ou qui, l'affectant dans l"un de ses éléments constitutifs ou l'un de ses élérments d'écuipement, le rendent impropre à sa clestination.

Une telle responsabilité n'a point lieu si le construc teur prouve que les dommages proviennent d'une cause étrangère.

Art. 2. Il est ajouté, après l'article 1792 du code civil. six articles 1792-1, 1792-2, 1792-3, 1792-4, 1792-5 et 1792-6 ainsi rédigés :

Art. 1792-1. Est réputé constructeur de l'ouvrage:

1) Tout architecte, entrepreneur, technicien ou autre personne liée au maître de l'ouvrage par un contrat de louage d"ouvtage :

2) Toute personne qui vend, après achèvement, un ouvrage quelle a construit ou fait construire :

3) Toute personne quj, bien qu'agissant en qualité de mandataire du propriétaire de l'ouvrage, accomplit une mission assimilable à celle d'un locateur d'ouvage.

Ar!. 1792-6. La réception est l'acte par lequel ]a maitre de l'ouvrage déclare accepter l'ouvrage avec ou sans résemes. Elle intervient à la demande de la partie la plus diligente, solt à l'amiable, soit a défaut judjciairement. Elle est, en tout état de cause, prononcée contradictoirement

La garantie de parfait achèvement, à laquelle l'entrepreneur est teru pendant un délai d'un an, à compter de la réception, s'étend à la réparation de tous les désordres signalés par lé maitre de l’ouvrage, soit au moyen de réserves mentionnées au procès-verbal de réception, soit par vole de notification écrite pour ceux révélés postérieurerient à la réception.

Art. 3. L'article 2270 du code civil est remplacè par les dispositions suivantes :

Art 2270. Toute personne physique ou morale dont la responsabilité peut être engagée en vertu des articles 1792 à 1792-4 du présent code est déchargée des responsabilités et garanties pesant suź elle, en application, des articles 1792 a 1792-2, aprés dix ans à compter de la réception des travaux ou, en application de l'article 1792-3, à ]'expiration du délai visé à cet article.

Art. 12. Le titre IV du livre II du code des assurances est remplacé par les dispositions suivantes

Art. L. 241-1. Toute personne physique ou morale. dont la responsabilité peut être engagée sur le fondement de la présomption établie par les articles 1792 et suivants du code civil à propos de travaux de bâtiment, doil ètre cotverte par une assurance. 


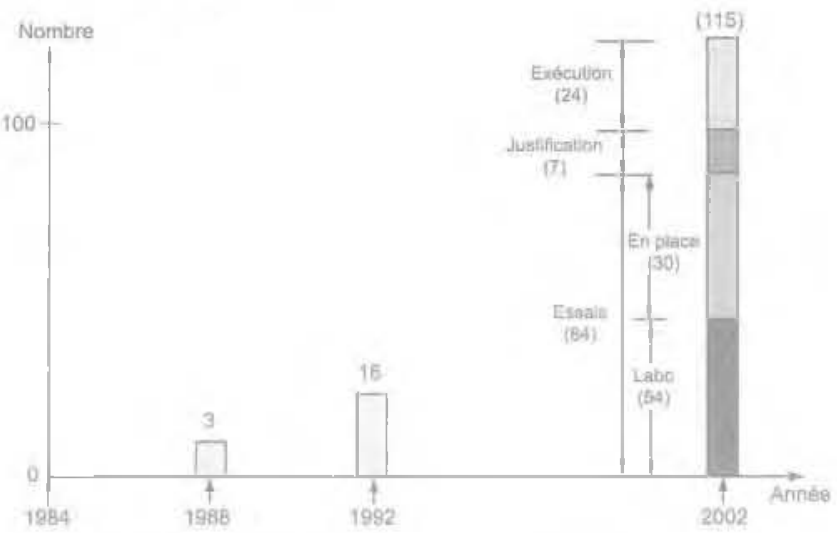

Évolution du nombre de nornes francaises applicaloles à la géotechnique de 1984 à 2002 (hors normes relatives aux gẻotextiles [41 en 2002), géomembranes (17) et granulats [86̈l, d'aprés Magnan, 2003)

\section{A.4}

\section{Extrait de la norme NF P94-500 révisée en 2006}

\section{Classification et enchaînement des missions types d'ingénierie géotechnique}

Tout ouvrage est en interaction avec son environnement géotechnique. C'est pourçuol, au même titre que les autres ingénieries, l'ingênierie géotechnique est une composante de la maitrise d'ceuve indispensable à l'étude puis à la réalisation de tout projet.
Le modéle géologique et le contexte qéotechnique général d'un site, définis lors d'une mission géotechnique préliminaire, ne peuvent servir qu’ả identifier des lisques potenliels liés aux aléas yéologiques du site. L'étude de leurs conséquences et leur réduction eventuelle ne peuvent être faites que lors d"une mission géotechnique au stade de la mise au point du projet : en effet, les contrantes géotechnigues de site sont conditionnées par la nature de l'ouvrage et variables dans le temps, puisque les formations géologiques se comportent différemment en fonction des sollicitations auxquelles elles sont soumises foéométrie de l'ouvrage, intensité et curée des efforts, cycles climatiques, procédés de construction, phasage des travalix notamment].

L'ingénierie géotechnicque doit donc être assoclée aux autres ingénieries, à toutes les êtapes successives G'étude et de réalisation d'un projet, et ainsi cortuibuer à une gestion efficace des risques géologiques afin de fiabiliser le délaj d'exécution, le coủt réel et la qualité des ouvrages géotechniques que comporte le projet.

L’enchaînement et la définition synthétique des missions types d"ingénierie géotechnique sont donnés dars les tableaux 1 et 2 . Les éléments de chaque mission sont spécifiès dans les chapitres 7 à 9 . Les exigences qui y sont présentées sont è respecter pour chacune des missions, en plus des exigences générales décrites au chapitre 5 de la présente norme. L'objectif de chaque mission, ainsi que ses limites, sont rappelés en tête de chaque chapitre. Les éléments de la prestation dinvestigations géotechniques sont spécifiés au chapitre 6.

TABLEAU I Schéma d'enchâ̂nement des missions types d'ingénierié géotechniqque.

\begin{tabular}{|c|c|c|c|c|}
\hline Etapen & $\begin{array}{l}\text { Phase d'avancement } \\
\text { du projet }\end{array}$ & Missions d'ingênierne géotechrique & $\begin{array}{l}\text { Objectlfs en termes } \\
\text { de gestion des risques liés } \\
\text { aus alóas géologiques }\end{array}$ & $\begin{array}{l}\text { Prestations d investiga- } \\
\text { tions gếrecirniques " }\end{array}$ \\
\hline \multirow[b]{2}{*}{1} & $\begin{array}{l}\text { Elude préliminaire } \\
\text { Eltude d'esquisse }\end{array}$ & $\begin{array}{l}\text { Etude géotechnigue préliminaire } \\
\text { de site }\{\mathrm{G} 11\}\end{array}$ & $\begin{array}{l}\text { PTemière identification des } \\
\text { risques }\end{array}$ & $\begin{array}{l}\text { Fonction des données } \\
\text { existantes }\end{array}$ \\
\hline & Avant-projet & $\begin{array}{l}\text { Étude gévtechrigue d'avant-projet } \\
\text { \{G:2\} }\end{array}$ & $\begin{array}{l}\text { Identification des aléas } \\
\text { majeurs et principes } \\
\text { generaux pour en timiter } \\
\text { les consequences }\end{array}$ & $\begin{array}{l}\text { Fonction des données } \\
\text { existantes et de l'avant- } \\
\text { projet }\end{array}$ \\
\hline 2 & $\begin{array}{l}\text { Projet } \\
\text { Assistance aux } \\
\text { Contrats de Travaux } \\
\text { (ACT) }\end{array}$ & Étude géotechnique de projer (G2) & $\begin{array}{l}\text { Identification des aléas } \\
\text { importants et dispositions } \\
\text { pour en réduire les } \\
\text { consequences }\end{array}$ & $\begin{array}{l}\text { Fonction des thoix } \\
\text { constructifs }\end{array}$ \\
\hline \multirow{2}{*}{3} & \multirow{2}{*}{ Exécution } & $\begin{array}{l}\text { Étude ef suivi géotechniques d’exécu- } \\
\text { ton (G3) }\end{array}$ & \multirow{2}{*}{$\begin{array}{l}\text { Identification des aléas } \\
\text { résiduels et dispositions } \\
\text { pour en limiter les consé- } \\
\text { quences }\end{array}$} & $\begin{array}{l}\text { Fonction des méthodes } \\
\text { de construction mises } \\
\text { en œuvre }\end{array}$ \\
\hline & & $\begin{array}{l}\text { Supervision géotechnique d'exéct- } \\
\text { tion (GA) }\end{array}$ & & $\begin{array}{l}\text { Fonction des conditions } \\
\text { rencontrées à l'exécu- } \\
\text { tion }\end{array}$ \\
\hline Cas particulier & $\begin{array}{l}\text { Etude d'ur ou } \\
\text { plusieurs élements } \\
\text { gétechniques spé- } \\
\text { cifiques }\end{array}$ & Diagnostic geutechnique (G5) & $\begin{array}{l}\text { Analyse des risqués liés } \\
\text { a ce ou ces éléments géd- } \\
\text { techniques }\end{array}$ & $\begin{array}{l}\text { Fonction de la } \\
\text { spécificité des édéments } \\
\text { étudiés }\end{array}$ \\
\hline
\end{tabular}

- Note. A définir per lingentarie gcotednique thargée de la misgibn comespondarie. 
tasteau II Classification des missions types d’ingénierje qéotechnique.

L'enchainement des missions d’ingénterie géotechnique doit suive les étapes d'élaboration et de réalisation de tout projet pour contribuer à la maîtrise des risques géologiques. Chaque mission s'appuie sur des investications géolechniques spécifigues. Il appartient au maître douvrage ou à son mandataire de veiller à la réalisation. successive de toutes ces missions par une ingénierie géotechniq̨ue.

\begin{tabular}{|c|c|c|}
\hline ape & & \\
\hline \multirow{3}{*}{1} & $\begin{array}{l}\text { ETUDES } \\
\text { GEOTECHNIQUES } \\
\text { PREALABLES [GI }\end{array}$ &  \\
\hline & $\begin{array}{l}\text { ETUDE } \\
\text { GEOTECHNIQUE } \\
\text { PRELIMINAIRE } \\
\text { DE SITE (G1T) }\end{array}$ & 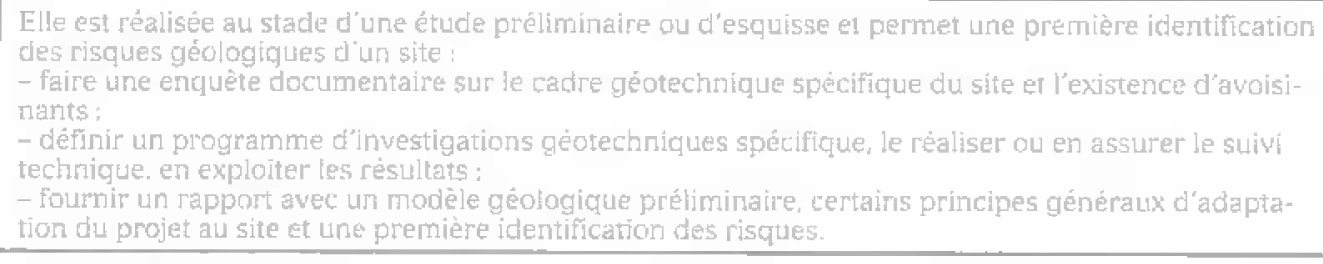 \\
\hline & $\begin{array}{l}\text { ÉTUDE } \\
\text { GEOTECHNIQUE } \\
\text { DAYANT-PROJET } \\
\text { (CI2) }\end{array}$ & 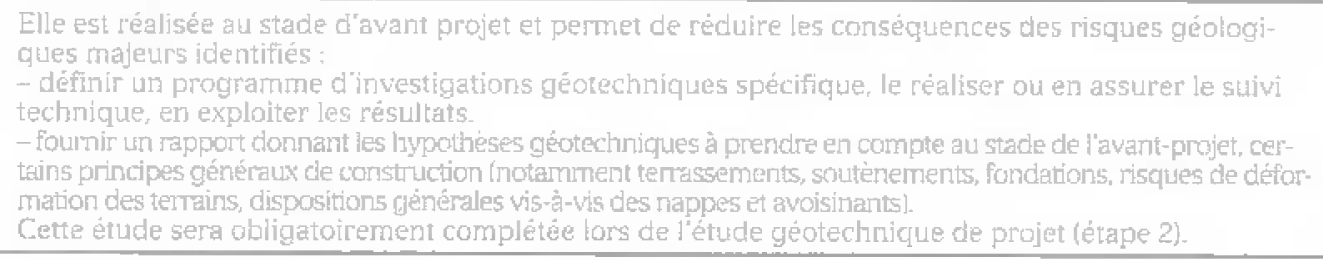 \\
\hline 2 & $\begin{array}{l}\text { ÉTUDE } \\
\text { GEOTECHNIQUE } \\
\text { DE PROHET [GZ) }\end{array}$ & 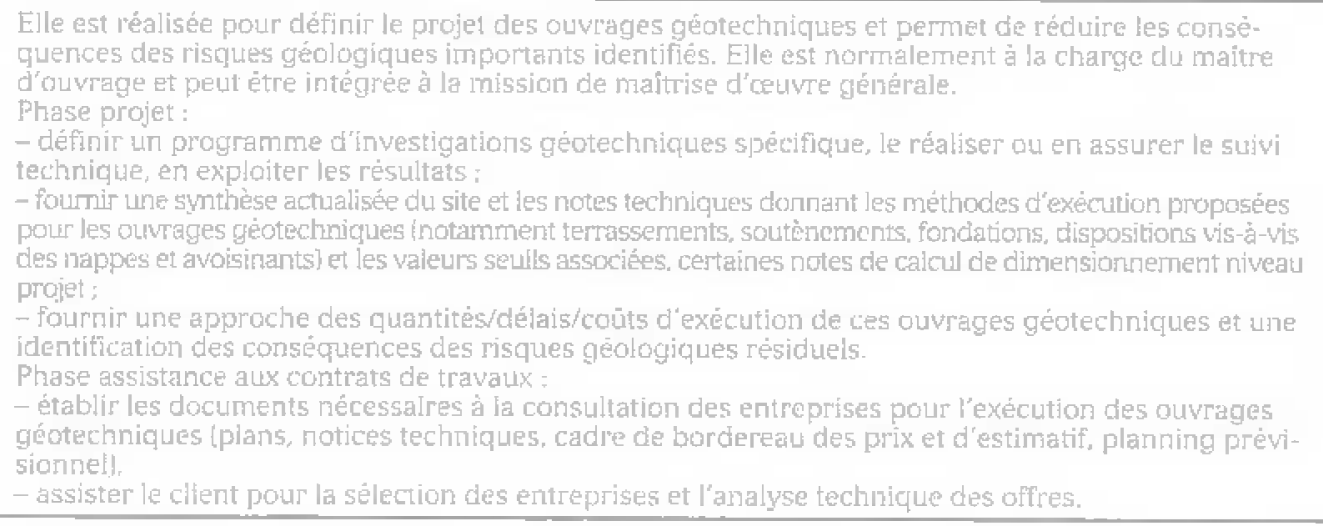 \\
\hline 3 & $\begin{array}{l}\text { EXECUTION } \\
\text { DES OUVRAGES } \\
\text { GEOTECHNIQUES } \\
\text { (G3 Et G4, distinctes } \\
\text { et simultanes) } \\
\text { ETUDE ET SUIV] } \\
\text { GEOTECHNIQUES } \\
\text { DEXECUTION (G3) }\end{array}$ & 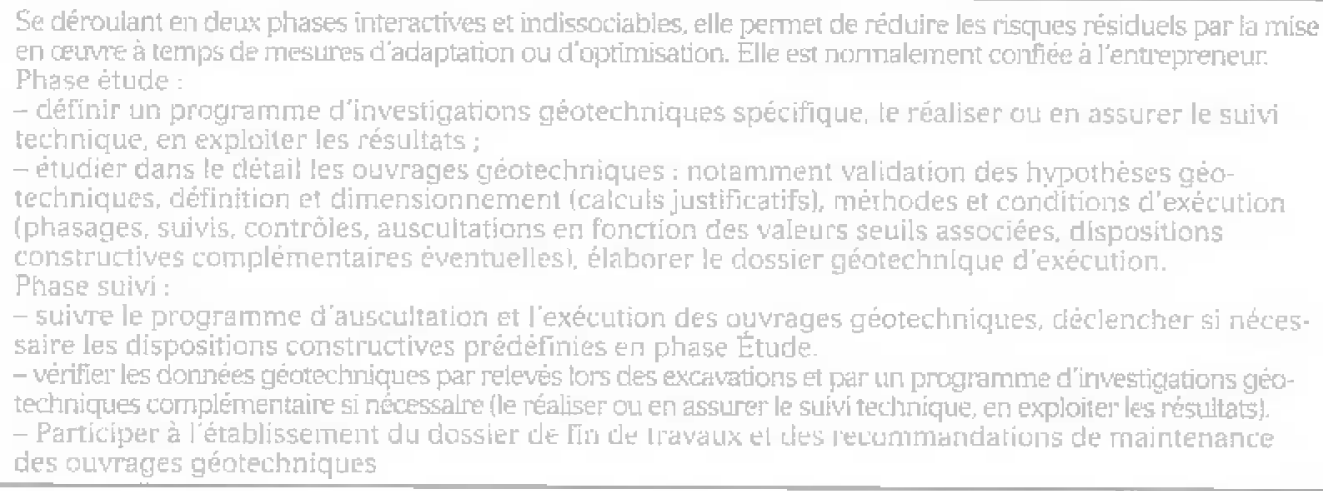 \\
\hline \multirow{2}{*}{ 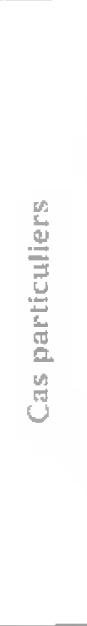 } & $\begin{array}{l}\text { SUPERVISION } \\
\text { GEOTECHWIQUE } \\
\text { D'EXECUTION (Gq) }\end{array}$ &  \\
\hline & $\begin{array}{l}\text { DIAGNOSTIC } \\
\text { GEOTECHNIQUE } \\
\text { [G5] }\end{array}$ & 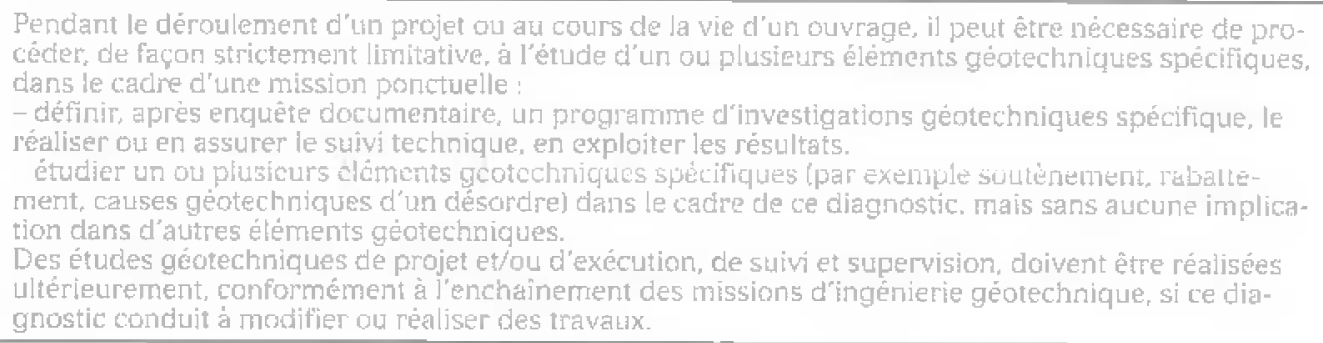 \\
\hline
\end{tabular}




\section{Conditions générales des missions d'ingénierie géotechnique (établies par l'Union syndicale géotechnique)}

\section{A.5.1}

\section{Cadre de la mission}

Par référence à la nome NF P gu-500 sur les misslons d"ingénierie géotechnique len particulier extrait de deux pages du chapitre 4 joint à toute offê et à tout rapport), il appartient au maitre d"ouvTage et à son maître d'culvre de veiller à ce que toutes les missions d'ingénierie géotechnique nécessaires à la conception puis à l'exécution de l'ouvrage solent engagées avec les moyens opportuns et confiées à des hommes de l"Alt.

L'enchaînement des missions d’ingénierie géotechnigue suit la succession des phases d'élaboration du projet, chacune de ces missions ne colvrant qu'tun donaine spécifigue de la conception ou de l'exécutior. En particulier :

- les missions d'étude géotechnique préliminaire de site (G11), d'étude géotechnique d"avant-projet (G12), d'étude géotechnique de projet (G2), d'étude et suivi géotechniques d'exécution (G3), de supervision géotechnique d'exécution (GA) sont réalisées dans l'ordre successif :

- exceptionnellement, une mission confiée à notre société peut ne contenir qu'une partie des prestations décrites dans la mission type correspondante après accord explicite, le client confiant obligatoirement le complément de la mission à un autre prestataire spécialisé en ingénierie géotechnique ;

- l'exécution d'investigations géotechnicues engage notre société uniquement sur la conformité des travaux exécutés à ceux contractuellement commandés et sur l'exactitude des résultals qu'elle fournit:

- toute mission d’ingénjerie géotechnique n'engage notre societé sur son devoir de consell que dans le cadre strict, dune patt, des objectifs explicitement définis dans notre proposition technique sur la base de lacueile la commande et ses avenants éventuels ont été établis, d'autre part, du projet du client décrit par les documents graphiques ou plans cités dans le rapport:

- toute mission d'étude géotechnique préliminaire de site, d'étude géotechnique d"avant projet ou de diagrostic géotechnique exclut lout engagement de notre société sur les quantités, cotits et délais d'exécution des futurs ouvrages géotechniques. De convention expresse, la responsabilité de notre société ne peut être engagée que dans I'hypothèse oü la mission suivante d'étude géotechnique de projet lui est confiée:

- une mission d'étude géotechnique de projet G2 engage notre société en tant qu'assistant technique à la maitrise d'cruvre dans les limites du contrat fixant l'étendue de la mission at la (ou les) partie(s) d'ouvtage(s) concemế(s).

La responsabilitë de notre société ne saurait être engagèe en dehors du cadre de la mission d'ingénierie géotechnicue objet du rapport. En particulier. toute modification apportée au projet ou à son environnement nécessite la réactualisation du rapport géotechnique dans le cadre d'une nouvelle mission.

\section{Recommandations}

11 est précisé que l'étude géotechnique l'epose sur une investigation du sol dont la malle ne pernet pas de lever la totalité des a héas toujours possibles en milieu naturel. En effet, des hétérogénéités, naturelles ou du fait de l'homme, des discontinuités et des aléas d'exécution peuvent apparaitre compte tenu du rapport entre le volume échantillonné ou testé et le volume soilicitåk par l'ouvrage, et ce d'autant plus que ces singularités éventuelles peuvent être limitées en extersion. Les éléments géotechniques nouveaux mis en évidence lors de l'exécution, pouvant avoir une influence sur les conclusions du rapport, dotvent immédiatement être signalés à l'ingénierie géotechnique chargée de l'étude et suivi géotechniques d'exécution (mission G3) afin qu'elle en aralyse les conséquences sur les conditions d"exécution. voire la conception de l'ouwrage géotechnique.

Si un caractère évolutif particulier a été mis en lumière (notamment glissement, érosion, dissolution. remblais évolutifs, tourbe), l'application des recommandations du rapport nécessite une validation à chaque étape suivante de la conception ou de l'exécution. En effet, un tel caractère évolutif peut remettre erı cause ces recommanciations, notamment s'il s'écoule un laps de temps important avant leur mise en ceutre.

\section{A.5.3}

\section{Rapport de la mission}

Le rapport géotechnique constitue le compte-rendu de lá mission d'ingénierie géotechrique définie par la commande au titre de laquelle il a été établi et dont les références sont rappelées en tête. A défaut de clauses spécifiques contractueles, la remise du rapport géotechnique fixe la fin de la mission.

Un rapport géotechnique et toutes ses amnexes identifiées constituent un ensemble indissociable. Les deux exemplaires de référence en sont les deux originaux conservés : un par le client et le second par notre société. Dans ce cadre, toute autre interprétation. qui pourrait être faite d'une communication ou reproduction partielle ne saurait engager la responsabilité de notre société. En particulier, l'utilisation même partielle de ces résultats et conclusions par un autre maitre d'ouvrage ou par un autie constructeur ou pour un autre ouvrage que celui objet de la mission confièe ne pourra en aucun cas engager la responsabilité de notre société et poutra entrainer des poursuites judiciaires.

\section{A.6}

\section{Inspection générale des finances et conseil général des Ponts et Chaussées}

Rapport sur l'assurance construction

Etabli par

Philippe DUMAS, inspecteur général des Finances

Alexandre MACAIRE, inspecteur des Finances

Jean-Jacques BRYAS, ingénieur général des Ponts et Cliaussées 
Jacques FRIGGIT, ingénieur en chef des Ponts et Chaussées

Isabelle VAULONT, inspectrice gènérale de l'Équipement

\section{Résumé}

la mission a établi, au terne de ses travaux, deux documents distincts : le présent rapport de synthèse et un rapport particulier, présenté en un fascicule séparé, qui compare les régimes d'assurance construction dans une vingtaine de pays étrangers.

Le rappont particulier met en évidence que le dispositif adopté par la France en matière d"assurance construction, établi essentiellement sur base législative, est l'un des plus régulés au monde s"agissant tant de la responsabilité des constructeurs à l'égard des maîtres d'ouvrage que de sa couverture par des mécanismes assuranciels ou de garantie. Il est également l'un des plus protecteurs pour les maitres d'ouvrage.

Le présent rapport de synthèse constate quant à lui, en sa premiere partie, que l'on est parvenu, aprés une longue phase de montée en régime, z̀ une relative stabilisation du dispositif : d'ure part, la sinistialité de la construction, guoique mal connue, ne semble pas augmenter : d'autre part, après de fortes hausses des primes, les régimes d'assurance construction sont désormais proches de l'équilibre comptable.

Le rapport souligne cependant plusieurs difficultés. D'une part, l'évaluation du dispositif se heurte ä un mangue d'infomation. s'agissant notamment de la sinistralité. D'autre part, les effets déresponsabilisants propres à tout mécanisme assuranciel sont aggravés par l'esprit, les règles et même le pratique du dispositif, qui conduisent, lors de la manifestation des sinistres, à impliquer au plus tôt I'assureur Do ${ }^{\text {is }}$, même lorsque le constmucteur responsable peut être clairement tdentifié et pourrait (voire cevrait, au titre de la garantie de parfait achèvementl réparer rapidement le dommage. Cet enclenchement rapide de la mécanique assurancielle a engendré des coùts élevés. Les mesures prises pour les réduire, via notamment la CRAC(b) aboutissent ả ce que les assureurs DO n'exercent que très partiellement les recours auxquels ils auraient droit contre les constructeurs responsables. Il en découle que, bien. souvent, les dommages causés par ces cerniers ne sont pas enregistrés dans leur historique cle siristralité et n'affectent donc pas leurs primes d"assurance les dommages dont ils sont responsables demeurent ainsi sans conséquence financiere pour eux; bien au contraire, leur réparation leur procure Lin chiffre d”atfaires supplêmentaire. La régulation économique de la sinistralité śen trouve ainsi sérieusement affectée. La possibilité d'un recour's au Bureau central de talrification pour obtenir une couverture que les assureurs sollicités ont préalablement refusée ne concount sans doute pas non plus à encourager du côté des constructeurs les comportements de prevention.

Par aflleurs, la réforme de 1978 appliquait un cadre juridique unique à un éventail de situations extrêmement différenciées, allant de la construction d'une seule maison, en lots séparés, par un particulier choisissant éventuellement de réaliser lui-même une partie du second oeuvre, jusqu"à de trés grands chantiers

(5) Dommage ouvrage.

16) Convention de reglement de l'assurance construction. décidés et financés par des maitres d'ouvtage rui snnt en pratique des professionnels expérimentés. L'expérience a montré les limites de cette réponse unique principalement aux deux extrémités de ce spectre se sont développés, par choix, par méconnaissance ou par nécessité, des écarts fréquents et peц satisfaisants entre le droit (une obligation légale d'assurance) et la pratique (une absence d'assurance ou une assurance partielle). Ainsi, les particuliers cui font construire sens $\mathrm{CM}^{|i|}$ ne souscrivent que très rarement une ADO'b! principalenent en raison de son coût, il est vrai particulièement élevé clans leur cas. Quant à la couverture assurancielie de la ACD ${ }^{19}$ des constructeurs cui interviennent sur les grainds chantiers, elle est souvent, notamment en raison des plafonds de capacité du marchê de la rêassurance, inférieure à l'étendue de leur responsabilité légale.

Enfin, le coût de l'assurance, plus élevé cư l'ätranger. atteint parfois des niveaux écononiquement dissuasifs.

La comparaison internationale effectuée par" la mis. Sion met en évidence que les dispositifs appliqués dans les autres pays sont extrêmement variés. Dans certains domaines (tels que la transparence), ils ne fournissent pas - sauf exception - de meilleurs résultats. Quelques a bonnes pratiques n émergent néanmoins : la primauté accordée, surtout dans les premières années de la vie de l'ouvrage, à la réparation directe par les constructeurs plutôt qu'a l'indemnisation assurancielle, la place accordée dans ce contexte à des procédures de conciliation, voire l'effet souvent responsabilisant à l'égard des constructeurs eux-nêmes de labsence d'obligation d'assurer qui existe de facto sinon de jure du côté des assureurs.

A partir de ce constat, la mission présente dans la deuxième partie du présent rapport de synthèse un ensemble de recomnandations de portée générale visant à amélioner la transparence, à réduire la sinistralité et à conforter les régines de responsabilité.

S'agissant de la transparence dv dispositif, la mission ne pouvait, dans le délaj de quelques mois dont elle a disposé, combler par elle-même le déficit d’information qu'elle a constaté. La constitution de bases de données, la réalisation d'études par échantillonnage et leur exploitation sont des tâches de longue diurée. La mission a pris connaissance des récentes décisions de l'Agence Qualité Construction (AOC) en liaison notamment avec les assureurs et les experts visant à améliorer tant l'alimentation que l'exploitation de ses propres bases de donnés. Elle pense que ces décisions pewvent atteindre leur objectif. Elles pourraient être utilement complétées par des études statistiques par échantillonnage. Si néanmoins ta voie du consenslis professionnel n'aboutissait pas, Ja collectivité serait Fondée à intervenir.

Outre l'amélioration de l'information sur la siristralité, la mission propose par ailleurs plusieurs mesures visant à permetre une meilleure transparence des couvertures assurancielles des constructeurs.

Elle recommande enfin que le Bureau central de tarifica tion (BCT) établisse et publie un rapport annuel d'activité

S'agissant de la réduction de la sinistralité, cet objectif devrait êtré atteint grâce à l"ensemble cies mesures recommandées par la mission, et au premier chef par

(7) Constructeu de maison individuelle as sens de la loi de 1900 .

(8) Assurance donmage ouvrage

(9) Responsabilité civile décennale. 
Celles visant à amélincer la transnarence de l'information et à responsabiliser les acteurs.

La mission préconise par ailleurs des mesures complémentaires visant a faciliter l'adaptation des fondations à la nature des sols.

La mission recommande enfin que les assureurs fran çís let, le cas échéant, les autres professionnels intéres sés\} étudient de facon plus détaillée qu'elle n'a pu eliemême le faire le dispositif dinspection systematioue par le National House-Building Council (NHBC) des entreprises et des chantiers ét évaluent la réduction du cout de l'assurance construction que pourrait engendrer lá mise en place d'un dispositif et de procédures analogues adap. tés aux structures françaises et au droit français, dispositif qui ne devrait pas interférer avec l'exercice des missions et des responsabilités des constructeurs ${ }^{10}$.

La mission suggère que cette étude, si elle est entreргіse, consacre une attention soutenue à la w valeut ajoutée y particulière qui doit normalement être celle des inspections du NHBC en ce qui concerne les qualifications professionneles des constucteurs britanniques adhérant au systènte et apprécie les enseignements que pourraient en tíer les assureurs français quant à leurs propres méthodes d'évaluation des l'isques.

S'agissant des régimes de responsabilité, la mission recommande pour l'essentiel de maintenir inchangé le régime de responsabilité décennale, exception faite du cas des EPERSI"廿.

En revanche, pour favoriser une réparation directe des sinistres sans implication de l'assurance, la mission. recommande de porter à deux ans la durée de la garantie de parfait achevement.

Par ailleurs, il lui est apparu soulaitable que, lorsque des particuliers recourent, en matière de construction, aux services d'intermédiaires professionnels, ces derniers puissent jouer dans tous les cas le rôle d"un " interlocuteur unique pauquel les riat tres d'ouvrage devraient, en cas de problème, pouvoir s'adresser par priorité (cf. infra) avant de saisir l'assureur DO, et sans avoir à faire appel aux différentes entreprises. La mission recommande pour cela dimpliquer davantage les promoteurs au sens larget ${ }^{\prime 21}$ dans la mise en oeuvre de la garantie de parfait achèvement et de faire bénéficier les acquéreurs successifs d'un imnetible pendant les deux ans suivant la prise de possession d'une protection d'effet équivalent à la GPA due au maitre d'ouvage.

I.a troisieme partie du rapport presente des recommandations spécifiques concernant les régimes d"assurance obligatoires, visant : $1^{\circ}$ à les rendre plus responsabilisants, $2^{\circ}$ à en rendre l'application effective plus confonne au droit, fut-ce au prix de certaines adaptations

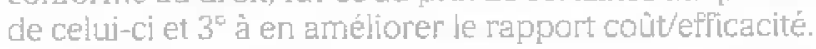

Ces recommandations, pour la plupat. visent à apporter des réponses différenciées à la grande variété des problèmes à traiter : petits et grands sinistres, peties et grands chantiers, petits et grands maittres d'ouvrage, etc.

(10) De tellos inspections pourraient notamment ẻtre organistecs par les assureurs lorsque aucuhe intervention d'un controleur techrique agrée n'a été plévue ou qu'aucune mission équivalente a une mission de base letle trise d"auve professionnelle par le maitre d'ouvrage.

(11) Eléments pouvant entraìner la responsabilite solidare.

(12) Promoteur immobller mais aussi vendeur d'immeuble á construire et vendeur professionnel d'immeuble acheve deguis moins de deux ans.
La mission préconise tout d'abord de privilécier la réparation directe des défauts de construction par les constructeurs eux-mêmes (au titre de la garantia de parfait achèvement et/ou de leur responsabilité décennale) plutôt que l'indemnisation assurancielle, et pour cela de rendre obligatoire la saisine par le maitre d'ouvrage ou l'acquéreur du bien de l'intermédiarre unique interposé dans sa construction ou cans sa vente (CM] ou promoteur) ou. à défaut, d'un constructeur pouvant raisonnablement être supposé responsable du problème, préalablement à celle de l'assureur.

Ce dernier ne serait donc appelé à n'intervenit financièmenent que si la saisine de l'intermédiaire / constructeur r’avait pas permis, dans un délai fixé, d'obtentr la réparation du dommage.

La mission préconise également l'introduction, en cas d'échec de cette saisine, d'une phase de conciliation préalable au déclenchement de la procédure assurancielle.

Ces deux mesures, associées au renforcement de la garantie de parfait achevement susmentionné, devraient faire sortir du champ de l'assutance un grand nombre de petits sinistres. Le coüt de l'assurance DO clevrait s'en trouver sensiblement réduit.

La mission estime que les assureurs sauront, par une tarification appropriée des primes de l'assurance RCD. inciter les constucteurs, promoteurs et CMI notamment, á réparer ou faire réparer directement el rapidement une grande partie des petits dommages. Les constructeurs seront ainsi plus efficacement encouragés à réduire la sinistralité résultant de leurs interventions.

Dans le cas des particuliers faisant construire sans CMI, la mission recommande que le contrat du lot de gros oeuve mentionne obligatolrement. comme le CMl, la référence de l'assurance de dommages souscrite par le maitre d'ouvrace (ou, sur mandat de sa part et pour son compte, par le litulaire dudit contrat).

Elle recommande par ailleurs de réduire l'obligation d'assurance de ces riaîtres d'ouvage à une ADO " de base \%, limitée aux atteintes à la solidité de l'ouvrage. Ces particuliers, actuellement dépolarvus d'ADO le plus souvent, seraient donc soumis ane obligation d'assurance certes plus limitée qu'actuellement mais beaucoup moins coûteuse et effectivement applicquée. Ils seraient bien sûn libres de souscrire, en sus, des couvertures assurancielles facultatives portant notamment sur l'impropriété à destination

S'agissant des grands chantiers - plus de 30 Me (qui, pour l'essentiel, ne portent pas sur des logements) et encore plus des très grands chantiers - plus de $150 \mathrm{M€}$-, la mission recontrande, er dehors du secteur du logement qui resterait soumis aux règles actuelles. de plafonner les garanties dues par l'assurance RCD ${ }^{[13 \mid}$ obligatoire et de laisser aux maîtres d'ouvrage le soin d'ajuster la couverture assurancielle exigée de chaque constructeum.

Utilisees par des rnaitres d'ouvrage avertis faisant un usage approprié des polices complémentaires de groupe à hauteur de la couverture obligatoire ou même d'une couverture complementaire facultative, ces dispositions clevraient résoudre une grande partia des difficultếs actuellement rencontrëes par" les constructeurs lorsqu'ils tentent de satisfaire à leur obligation d'assurance sans restreindre l'accès des PME aux marchés de construction.

(13) Pesponsabilité civile décennate. 
I.a mission recommande enalament gue snjent précisées les conditions dans lesquelles les maittes d'ouvrage publics peuvent ou pourratent, dans ce contexte, recourir à des polices complémentaires de groupe et que, simultanément, ces derniers soient incités à déteminer et à expliciter la politique assurancielle qu'ils souhaitent suivre.

Pal ailleurs, hors logement, la nission recommande d'élargir le champ des exonérations de lobilgation d"ADO. voire de supprimer cette obligation, pour restaurer la liberté contractuelle la ot sa restriction n"est pas justifiec..

S'agissant du logement, dans les cas où un seul interlocuteur (CMI et promoteur pour l'essentiel) existe face au maître ouvrage, la mission recommancle gue soit étudiée la faisabilité du remplacenent de l'ADO et de la RCD par une assurance unique de responsabilité renforcée.

La mission constate qu'il n'a pas étế effectué jusqu'à présent d'étude évaluant spécifiquement la sinistralité des opérations assurées sur décision du BCI. Elle préconise donc qu'une telle étude soit condulte st permette ainsi au Burăa lui même de mielix apprécier la pertinence des tarifications appliquées par lui a! regard de l'mportance des risques.

La mission propose aussi de modifier les procédures de gestion de l'assurance construction afin de les rendre plus responsabilisantes et notamment d'aménager le fonctionnement de la CRAC aîn d"accroitre le taLx de recours des assureurs DO contre les assureurs ACD.

Elle recommande également d"autoriser la pratique de franchises en ADO, et de demander aux assurés ure participation aux coùts d'expertise en cas de déclaration abusive.

Enfin, la question célicate de l'impact éventuel des textes et projets européens sur le régine de l'assurance construction est abordée dans l'annexe 1.

En conclusion, la mission souligne cte la mise en oeuvre de ses recommandations, qui suppose pour certaines d'entre elles le passage par la vole législative. devrait être préparée par une phase de concertation avec les professionnels, au sein rotamment de la commission technique de l'assurance construction.

\section{Extraits du rapport}

\section{p. 17}

La mission a eu le souci d’essayer de répondre à la demande qui lui ètait laite de porter notamment une attention particulière a la typologie des sinisitus selon leur taille et leur origine ainsi gu" son évolution éventuelle $n$. Il lui était également demandé que certains vecteurs de sinistralitè tels que la guestion des fondations des maisons individuetes fassent lobjet d'un examen plus précis.

Mais elle náa pas, en délinitive, pu rassembler des informations détaillées sur ces points.

\subsubsection{Adéquation des fondations à la nature du sol}

Ĺattention de la mission a été à de nombreuses reprises attirée sur les dommages causés par une mauvaisc adćquation des fondations au sol.

Les données disponibles sur les bâtiments assurés - on rappelle qu'il s'agit de donnès portant sur les bâtiments assurés en $\mathrm{ADO}$ - montrent que les sinistres de ce type ne sont pas les plus fréguents't", mais qu'ils sont d'ur linon tant unitaire élevé. It s'agit donc d'une siristralite d'intonsité, non de fréguence. En tant que telle, elle ne devtait relever ni de l'auto-assurance, ni mêne du préfinancement par le proptlétaire lésé, surtout s'agissant des particuliers. La mission a pris connaissance de diverses propositions visant a rendre obligatoine une étude de sol avant toute construction lou meme avant toute vente de terrain à bátir), voire mème à renforcer les normes de fondation sur l'ensemble du territoine.

En premier lieu, elle rappelle le principe économique selon lequel be coht total des mesures préventives imposées par l'État ne doit pas, du moins en matière de donmages aux biens, être trop supérieurisi au montant des sinistres qu'elles permettent d'éviter.

La mise en oeuve de ce principe suppose une bonne connaissance non seulement du nombre, du montant unitaire et de la typologie des dommages ceusés par une mauvaise acéquation des fondations à la nature du sol, mais également de la proportion dans laquelle ces dommages pourratent être réduits, et avec quelle probabilitélat par les diverses mesures envisageables (i diagnostics sols y plus ou moins poussés. donc d'un coút plus ou moirs élevé, imposant la réali sation de fondations plus ou moins robustes donc plus ou moins chères, etc.).

Or cette connaissance fait actuellement détaut : la mission $\Omega^{\prime \prime a}$ pas et connaissance d'etude permettant de chiffrer ne serait-ce qu'approximativement le bilan économique des mesures proposées ${ }^{17 i}$ !

Une première étape est donc la realisation d'une telle étude. Elle est de la responsabilité de l'État, relavé éventuellement par l'AQG, en collaboration avec les professions concemées dans te cadre notamment de la commission technique de l'assurance construction (CTAC)

A supposer qu'au vu de cette êtude la collectivité impose des udiagnostics sol obligatoires, it conviendrait de clétertminer:

à quels acteurs du processus de construction elle l'imposeraitit's:

(14) Les sinistres les plus fréguents sont ceux provenant dthn defaut d'êtancheité.

(15) II peut luj ètue superrieur, nasis seulement dass une proportion

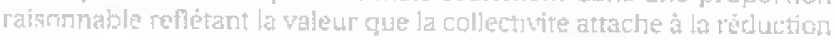
du risque apporte par la mesure.

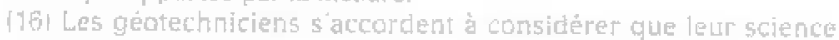
п"Est pas totatement exacte. Plus une elude géotechnique est pousgéte "par" augmentalion du nombre de sondages par exemplel, pilus ellé


elle me peut l'arnuler.

(17) Les bases de donnees de l'AQC sont insutisummern fiables rur sur exe point er les données émanant des assureurs al distinguent pas les sin'stres par cause

[18) La mission a bien noté que la CFEC ICompagnie francaise des axparts constructionl envisage de merer une telle étude. Seg membres sont bien placés, tant par leur position dans le mécanisme de l'assurance construction que par leurs competences teclunic|ues, pour collecter l'information nécessaire.

(19) Par exemple, á l'acheteuj ou au vendeur d"un telrair à bätir ? Le vendeur the connail pas a priori la construction envisagéc par les acheteur potentiels et est conc mal placé pour effectuer le diagnostic : si des sondages surl néceşares. I"acheteur ne peut efrectuer le diagnostic qu'avec l'autorisation da wendeut ; par ailleurs, si plusieurs acheteurs font effectuer ur diagnostic adapte a leur projet. puls renoncent l'un après l'autre, une dépense significative aura été effectues en pure perte. 
- quelle en serait l'étendue et qui en supporterait le cout (un diagnostic nécessitant des sondages pouvant coüter plusieurs milliets d'euros, soit un montant du même ordre de grandeur que l'avantage de taux procuré par le prét à taux zérol;

- au cas ou un sinistre surviendrat alors gue les conclusions du diagnostic ont été prises en considération (éventualité à ne pas écarter), qui supporterait la responsabilité d'éventıels dommages

- sur quel périmétre elle l’imposerait (plusieurs pérímètres différents êtan nécessaires si l'on veut traiter l'ensemble des risques liés á la présence d'arglle, au risque de tassement, etc.) ;

- dans quelles proportions la sinistralité causée par l'inadéquation des fondations au sol seralt réduite par ces ninesures.

Par ailleurs, la mission considere qu'en matèe de construction non residentielle, il appartient aux professionnels, entourés si nécessaire de conseils plus compétents, de gérer au mieux le risque de dommages á l'ouvrage pouvant découler" d'une inadéquation des fondations au sol: une éventuelle intervention de l'État au titre de l'assurance construction ${ }^{[20)}$ en ce domaine ne se justifierait qu’en matière de logement

S'agissant des logements construits par des maîtres d'ouvrage professionnels ou avec le concours de CMI, la pratique d'études de sol au noins sommaires semble en voie de généralisation, au vu des entretiens menés par la mission. Le problème se pose donc principalement (mais non exclusivement) dans le cas des particuliers quif font construire sans CMI. La mesure recommandée par la uission au $\$ 3,2$ devrait assujetti efficacement ces maitres d'ouvrage à une obligation d'ADO limitée aux atteintes à la soliclité de l'ouvrage. L'assureul" qui supportera le risque d"inadéquation des fondations à la rature du sol demandera sans doute, dans les cas ou le sol peut ètre présumé particulièrement risquét211, wne étude de sol ou un renforcement a priori des fondations'22!. Cela contribuera à une Tjeilleure gestion du risque de sol.

Enfin, la collectivité peut déla imposer des contraintes portant sur la nature des fondations des constructions, au tirle de l'articke R 111-2 du code de l'urbanismelaz et des plans de prevention des risques.

Ces contraintes seront elles-mêmes peut-être renforcées dans le futur si les pouvoirs publics retiennent tout ou partie des mesures préconisées par la récente mission interministérielle sur lè régine d"indemnisa. tion des victimes de catastrophes naturelles.

(20i [I en wa bien sum ditferemunent en matière de sécumlé des personnes. (21) Dans les autres cas, 11 mutualisera le risque.

1221 Les exigences des assureum Do en la matidre devlaient cependant demeurer raisonmałbles. En l"absence d'une telle étude, le BCI pourrait arguer de l'absence d'alé:a pour refuser au maitre d'auvrage le benéfice de l"obligation d’assurer. S'il savérait cepenchat que les exigences des assureur DO étrient excessives, le BTC pourrait étre

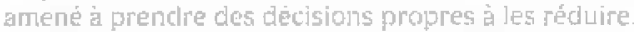

(23) Qui dispose que or le pernis de construire peut étre refusé ou n'être accorde que sous réserve de l'obser"wation de presctaptions


sont de nature à porter arteinte ta la salubrité ou ả la sécurité publiçue.

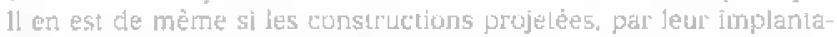
tion a proximilé diautres instalkations, leurs caractếristiques ou leur siruation, sont de nature à porter atteinte á la salubrifé ou à la sécurité publigue 2
Pour l'ensemble de ces raisons, la mission ${ }^{271}$ r'a pas. recommandé de rendre obligatoires de façon générale des a diagnostics sol m.

En revanche, dans sa majorité, la missiun a consjdéré qu'il pourrait être exigé que tout vendeư" de terrain à bâtir porte à la connaissance de l'acheteur les informations en sa possession ${ }^{251}$ sur les caractéristiques du sol et du sous-sol susceptibles à sa connaíssarce d'entrainer des inconvénients particuliers pour la réalisation d'ume construction, notamment en ce qui concerne l'irnportance des fondations ${ }^{[26]}$.

La mission a par ailleurs noté que certains constructeurs de maisons individuelles soukaiteraient, lomscue des fondations plus couteuses que prévu apparaissont nécessaines après la conclusion du contrat, pouvoir être déliés de leur engagement concernant la fixitê du prix.

Sur ce sujet, la mission a considéré que deux situations pouvaient en pratique se rencontrer.

Lorsque le particulier dispose délà du terrain sur lequel la maison qu'il désire sera construite, il appartient au CMI. s"il le fuge nécessaire, de demander à son client potentiel, de faire réaliser lui-même une étude de sol avant de fixer" son prix ou, s'il veut en prendre le risque commercial, de réaliser lui-même cette étude et de ne fixer sa proposition de prix quaprès son achèvement.

Larsque, au contraire, le CMI assiste le maitre d'ouvrage dans l'identification d'un terrain à bâtir, il n'est pas illégitime qu'il fasse procéder à ses frais aux études de sol nécessaires avant de s'engager sur un prix de vente et qu'à défaut le risque de sol demeure à sa charge, le CMI étant en situation, en tant que professionnel, de mutualiser ce risque, c'est-à-dire de le répartir sur tout un ensemble de constructions.

En conclusion, la mission ne considère pas culil soit utile d'apporter des aménagements au principe selon lequel les contrats de construction de maison individuelie sont conclus à prix ferme.

\subsection{Améliorer l'assurabilité de certains profes- sionnels}

On a souligné au \$1.3.2.3 les difficultés que rencontrent certains professionels a trouver une assurance RCD.

Elles sont aggravées par plusieurs difficultés propres au domaine de la construction.

Le jeu de la concurtence conduit souvent certains prestataires à prendre des marchés pour un montant d'honoraires trop lable ; il en résulte un risque élcvé de maluvaise réalisation des prestations. La résolution de cette difficulté relêve des prestataires eux-mêmes.

(24) Pac arablogit avec dos dispositions similaires existames. Lathicle L. 75-2-I du code minier jmpose au vendeur d'un terTain sup le trêfonds duquel une mine a eté exploitée d'en informer par êclit l'ac-


conraisse, des dangers ou inconvenients importants gui résultent de l'explotitaton w. La'ticle I. 125-5 du code de l'environnement impose au vendeur dun immeuble báti qui a subi un sinistre ayant donné lieu au wersement d"une indemnitè au titre des risques catastrophes naturelles ou technologicues, "d'informer par écrlt l'acquéreur de tout sinistre sumenu pendan: la période oit il a éte proprietaire de I’immeuble ou dont il a été luj-mème informé en application des pré-



(25) I" ne lui serait donc pes demandé de recher:ler des informations


diamostic soil reallsé.

(26) Dewraient également être fixees les sanctions qu'entrafnerait l'inuliservation de cette dispusition. 
Intsmue le Anmaine en question. tol gue la géntechnique, ne relève pas de la science exacte, il est difficile pour les professionnels de faire valoir l'imperfection. de leur science pour dégager leur responsabilité en cas de désordres, même sì des précautions rédactionnelles ont été prises lors de la définition de leur mission. Leur responsabilité, qui relève de l'appréciation des tribunaux sur le fondement des travaux des experts judiciaires, peut difficilement être réduite, à supposer qu'on le souhaite, par des mesures relevant du domaine de l'assurance. Par contre s'agissant du risque géotechnique celui-ci sera mieux evalué, et réduit, si le maître de l'ouvrage associe l'ingénierie géotechnique aux autres ingénieries à toutes les ètapes d'étude et de réalisation. du projet comme le préconise l'avant-projet de norme relatif aux missions d'ingérierie géotechnique quì vient d'être soumis à enquête probatoine (en vue d'être substituée à la nome homologuée NFP 94-500).

La bongévité des prestataires intellectuels, qui sont frẻquemment des personnes physiques, est souvent supérieure â celle des personnes morales qui exécutent les travaux; il est donc courant, lorsqu'un dommage survient, que les premiers exercent encore leur activité alors que les secondes ont disparu et alors que l'identité de leur assureur, tend de supporter le coút des dommages dont elles sont responsables, n'a pas été demandée ou conservée par le maitre d'ouvrage ou le prestataire intellectuel.

Compte tenu du fait que les tribunaux prononcent souvent des condamnations in solidum, une fraction élevée des dommages peut alor's être mise à la charge du prestataire

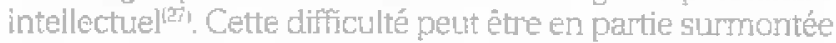
par la collecte et la conservation des attestations d'assurance des autres constucteurs participant aux chantiers.
Néanmnins, les difficultés que rencontrent certaines professions pour assurer leur responsabilité décennale trouvent principalement leur origine dans le plafond très élevé (égal au coût total de reconstruction de l'ouvrage) de la couverture imposée par la loj.

La mesure reconmandée au \$ 3.3 résoudrät en grande partie cette difficulté, puisqu'elle permettrait aux maîtres d“ouvrage recourant à une police complémentaire de groupe de différencier la couverture assurancielle exigée des différents constructeurs concourant à une mêne réalisation, et donc de l'adapter à l'offre disponible sur le marché de l'assurance pour chaque profession. Au-delà de cette couverture individualisée, la responsabilité globale de ces constructeurs serait couverte par la police complémentaire de groupe, dans le cadre d'une obligation de couverture jusqu'à un montant d'indemnités de 30 millions d'euros et d"une couverture facultative au-delà. Bien utilisée par les maîtres d'ouvrage, cette mesure devrait donc améliorer significativement l'assurabilité de certaines professions.

Par ailleurs, la mission a observé qu'à l'étranger (Royaume-Uni, Allemagne) la question de l'assurance des agences et entreprises nouvellement créées est parfois résolue dans le cadre de polices dont les organismes professionnels, notamment ceux qui recrutent leurs associes sur le fondement d'une procédure de qualification préalable, négocient les temes, au bénéfice de leurs adhérents, le cas êchéant après mise en concurrence de plusieur"s courtiers. Les professions dont les jeunes membres rencontrent en France des difficultés à s'assurer, pourraient sinspirer de ces exemples.

(27) Dans le même sans, la mission a relevé que le barème de préfinanceThent forfaitaire prévu par la CRAC impule parfois au prestataire intellectuel une fraction élvive de la responsabilite ; ce dernier peut ta contester" mais cela a un coût, au moins administutif. 